\title{
Changes 2006-2010 in the list of Finnish insects
}

\author{
Hans Silfverberg
}

Silfverberg, H. 2012: Changes 2006-2010 in the list of Finnish insects. — Entomol. Fennica 23: 18-41.

During the period 142 species have been deleted from the list of Finnish insects, and 1,223 species have been added to it. At the end of year 2010 a total of 21,613 insect species were known from Finland.

H. Silfverberg, Zoological Museum, P.O.Box 17, 00014 Helsingfors, Finland; Email: hans.silfverberg@helsinki.fi

Received 1 April 2011, accepted 18 June 2011

\section{Introduction}

This paper is a sequel to a similar list published in 2007 (Silfverberg, Entomol. Fenn. 18:82-101), which itself was a continuation of a series of lists published in 1921-2001 first by Hellén and then by Silfverberg. Insect species added to the fauna of Finland during the five-year period are listed, with references, as are those deleted from the fauna. A few previously unnoted changes have also been included.

In previous lists only data from printed publications have been included. Nowadays fairly much has been published in electronic form, and such data cannot be ignored. When referred to, such publications are listed in the beginning of each order.

For the new species the locality has generally been given, with its biogeographical province. If the species has been reported from many localities often only the provinces have been listed, or the abbreviation m.l. (= many localities) has been used. When Finland is mentioned as the locality, the original record gave no more exact location. Some other abbreviations or signs must also be explained. Thus f.d. = false determination and sp.dist. $=$ distinct species (previously considered a variety, a synonym or such like); in literature references $\mathrm{EF}=$ Entomologica Fennica and $\mathrm{NE}=$ Notulae Entomologicae.
A species enclosed in square brackets is considered accidentally imported, and has not been included in the species total. Again square brackets have in some cases been employed for the name used in the original record when different from the one used here. Other symbols are $*=$ new to science and $!=$ different from the check list mentioned for the order.

There were some counting errors in the previous list (Silfverberg 2007). Numbers in this list are based on corrected ones. During the period covered by this list there have been 126 species deleted from the Finnish fauna, and 1,223 species added to it. At the end of year 2010 a total of 21,617 insect species were known from Finland.

\section{Additions and deletions from the list of Finnish insects}

\section{Collembola}

Deletions

Folsomia diplophthalma (Ax.) (f.d., $=$ F. palaearctica Pot. \& Bab.). Fjellberg 2007 Fauna Ent. Scand. 42:38.

Vertagopus sarekensis (Wahlgr. ) (f.d.). ibid.:104, 252. Isotoma violacea Tullb. (f.d., = Desoria tolya Fjellb. \& D. hiemalis (Sch.)). ibid.:120, 122.

Sminthurides signatus (Krausb.) (f.d., = S. pseudassimilis Stach). ibid.:181. 
Arrhopalites pygmaeus (Wank.) (f.d.). ibid.:191, 254.

Sminthurus flaviceps Tullb. (f.d.). ibid.:226.

\section{Additions}

Ceratophysella engadinensis (Gis.) Ka: Kotka, $T b$ : Jyväskylä. Huhta et al. 2010 Memoranda Soc. Fauna Flora Fenn. 86:76.

Friesea truncata Cass. Al. ibid.:76.

Protaphorura tricampata (Gis.) $\mathrm{Tb}, \mathrm{Kb}, \mathrm{Ob}, \mathrm{Ks}$. ibid.:77.

Stenaphorura lubbocki Börn. N: Helsinki. ibid.:78.

Mesaphorura italica (Rus.) Ks: Kuusamo. ibid.:77.

- sylvatica (Rus.) $A b, N, K a, O a, T b, O b, K s, L k$. ibid.:77.

Wankeliella peterseni Rus. Ks: Kuusamo. ibid.:78.

Anurophorus palaearcticus Pot. Tb. Fjellberg 2007 Fauna Ent. Scand. 42:23, 250.

- atlanticus Fjellb. N, Oa. ibid.:24, 250.

Folsomia candida (Will.) N, Oa. Fjellberg 2007 ibid.:28, 250.

- bisetosa Gisin Lk. ibid.:32, 250.

- manolachei Bagn. Ab, Ks, Lk. ibid.:38, 250.

Proisotoma clavipila (Ax.) Ka, Oa, Ob, Lk. ibid.:81, 251.

Desoria grisea (Lubb.) Al, Ab, N, Ka, Oa, Kb, Ob. Huhta et al. 2010:76.

Isotomurus italicus Carap. Kb. Fjellberg 2007:94, 252.

* - graminis Fjellb. Ob. ibid.:96, 252.

- stuxbergi (Tullb.) Ab, Ta. ibid.:98, 252.

- antennalis Bagn. $N$ : Helsinki, Ka: Kotka. Huhta et al. 2010:77.

Vertagopus haagvari Fjellb. Ab, Ta, Tb. Fjellberg 2007:103, 252.

Parisotoma trichaetosa (Mart.) Ob: Oulu (V. Huhta). ibid.:111.

Isotoma riparia (Nic.) (m.1.). ibid.:115, 252.

- caerulea Bourl. (m.1.). ibid.:116, 252.

*Desoria tolya Fjellb. (m.1.). ibid.:120, 252.

* - potapovi Fjellb. Lk, Li. ibid.:126, 253.

Entomobrya multifasciata (Tullb.) (sp.dist, m.l.). ibid.:139, 253.

Lepidocyrtus pallidus Reut. (sp.dist.) $N, S a, K b$. ibid.: 147, 253.

Sminthurides parvulus (Krausb.) Tb. ibid.:184, 254.

Arrhopalites cochlearifer Gisin Ka, Ta. ibid.:189, 254.

Sminthurinus transversalis Ax. (sp.dist.) N, Om, Ob. ibid.:196, 255.

- signatus (Krausb.) (sp.dist.) (m.1.). ibid.:197, 255.

- alpinus Gis. N: Helsinki. Huhta et al. 2010:78.

Dicyrtomina flavosignata (Tullb.) Ab, N. Fjellberg 2007:206, 255.

Heterosminthurus claviger Gisin (m.1.). ibid.:213, 255.

Present number: $215-6+33=242$ spp.

\section{Ephemeroptera}

Deletions

Baetis tracheatus Keff. \& Mach. (f.d., = B. jaervii Savol.). Savolainen 2009a EF 20:185. Savolainen 2009b Kulumus 15:26.

Additions

*Baetis jaervii Savol. Ta, Kl, Tb, Sb, Kb, Om, Ok, Ks, $L k$, Li. Savolainen 2009a EF 20:183. Savolainen 2009b Kulumus 15:3,22.

Present number: $55-1+1=55 \mathrm{spp}$.

\section{Odonata}

Korentowiki. $(30.9 .2010)$

http://www.sudenkorento.fi/kwiki

Additions

Aeshna affinis V.d.Lind. N: Helsinki. Schröter \& Karjalainen 2009 Crenata 2:36.

Anax imperator (Leach) Al: Lågskär (P. Pynnönen). Korentowiki

Sympetrum pedemontanum (Alli.) N: Tvärminne (P. Ahlroth \& A. Albrecht) ibid.

Present number: $54+3=57$ spp.

\section{Psocoptera}

Kanervo, J. 2010. Suomen jäytiäiset / The Psocoptera of Finland. http://www.sci.utu.fi/projects/biologia/elainmuseo/hemi/psoc/psocmaps.htm (15.11.2010)

Additions

Liposcelis palatina Roesl. Ab: Lemu. Kanervo.

Peripsocus consobrinus Pearm. Ka: Virolahti. Kanervo.

Elipsocus moebiusi Tet. Ab, N, St, Ta, Ob, Ok. Kanervo.

Philotarsus parviceps Roesl. Al-Om. Kanervo.

Present number: $67+4=71$ spp.

\section{Hemiptera}

Hem1 - Hemiptera-työryhmä, Albrecht, A. Rinne, V. \& Hemiptera Expert Group. 2010. Suomen luteet. / The Heteroptera of Finland. http://www.sci.utu.fi/projects/biologia/elainmuseo/hemi/het/ludemaps.htm (25.11.2010)

Hem2 - Söderman, G., Albrecht, A., Rinne, V. \& Hemiptera Expert Group. 2010. Suomen kaskaat. / The Auchenorrhyncha of Finland. http://www.sci.utu.fi/projects/biologia/elainmuseo/hemi/auch/kaskmaps.htm (15.11.2010)

Hem3 - Mattila, K., Söderman, G., Albrecht, A., Rinne, V. \& Hemiptera Expert Group. 2010. Suomen kempit, 
jauhiaiset ja kilpikirvat. / The Psylloidea, Aleyrodoidea and Coccoidea of Finland. http://www.sci. utu.fi/projects/biologia/elainmuseo/hemi/psyll_etc/ kempmaps.htm (15.11.2010)

Hem4 - Albrecht, A. \& Hemiptera Expert Group. 2010. Suomen kirva-atlas. / Atlas över Finlands bladlöss / Atlas of the Aphids of Finland. http://www.luomus.fi/elaintiede/hyonteiset/tutkimus/kirvat/atlas.htm (15.6.2010)

Albrecht, A., Mattila, K., Rinne, V. \& Söderman, G. 2008. Suomen nivelkärsäisten luettelo. http://www.sci.utu. fi/projects/biologia/elainmuseo/hemi/tyoryhma/ Check-list_of_Finnish_Hemiptera.pdf (28.11.2008)

Rintala, T. \& Rinne, V. 2010. Suomen luteet. Tibiale Oy, Helsinki, 352 pp.

Deletions

Hydrometra stagnorum (L.) (dubious record). Albrecht et al. 2008:1.

Edwardsiana hippocastani (Edw.) (f.d., = E. ulmiphaga Wils. \& Cl.). Albrecht et al. 2006 Sahlbergia 11(2):9.

Macrosteles fascifrons (Stål) (= M. alpinus (Zett.)). Söderman 2007 The Finnish Environment 7:45.

Aphalara rumicicola Klim. (= A. polygoni Först.) Albrecht et al. 2008:17.

Aphis striata HRL (f.d.). Hem4

- praeterita Walk. (f.d.). ibid.

Toxopterella drepanosiphoides McGill. \& Br. (= Muscaphis escherichi (Börn.)). Remaudière \& Remaudière 1997 Catal. Aphid. monde, p. 123.

Additions

Glaenocorisa cavifrons (Thoms.) (sp.dist.) Ok-Li. Rintala \& Rinne 2010:42.

Plea minutissima Leach $N$ : Helsinki. Hem1.

Velia caprai Taman. Al: Eckerö. Rintala \& Rinne 2010:59.

Galeatus affinis (H.-Sch.) Finland. Albrecht et al. 2008:3

Stephanitis pyri (F.) Finland. ibid.:3.

- rhododendri Horv. N: Elimäki. Rintala \& Rinne 2010:79.

Macrolophus melanotoma (Costa) Finland. Albrecht et al. 2008:4.

Dicyphus errans (Wolff) Al. Rintala \& Rinne 2010:85.

- pallicornis (Fieb.) Ab: Tarvasjoki. Albrecht et al. 2006 Sahlbergia 11(2):3, Rintala \& Rinne 2010:85.

- epilobii Reut. $N$ : Ingå. Hem1.

Tupiocoris rhododendri (Doll.) N: Helsinki. Rintala \& Rinne 2010:87.

[Nesidiocoris tenuis (Reut.)] Oa. Huusela-Veistola et al. 2010 Kasvinsuojelulehti 43:20, Rintala \& Rinne 2010:267.

Deraeocoris lutescens (Schill.) Ab: Turku. Hem1.
Dichrooscytus gustavi Josif. Ta: Tampere (J. Kirjavainen). Albrecht et al. 2006:4, Rintala \& Rinne 2010:110.

Dichrooscytus intermedius Reut. $A b, N, T a$. Rintala \& Rinne 2010:111.

Acetropis gimmerthalii (Flor) Kl: Parikkala. Hem1.

Pseudoloxops coccineus (M.-D.) Al: Finström \& Mariehamn. Albrecht et al. 2006:4, Rintala \& Rinne 2010:135.

Psallus montanus (Jos.) N, Ka. Rintala \& Rinne 2010:266.

Chlamydatus drymophilus Vinok. Ab-Le. ibid.:148.

Amblytylys nasutus (Kirschb.) N: Tvärminne. Hem1.

Elatophilus nigrellus (Zett.) (sp. dist.) Ab-Ks. Rintala \& Rinne 2010:172.

Orius laticollis (Reut.) Al: Finström (P. Ahlroth). Albrecht et al. 2006:4, Rintala \& Rinne 2010:175.

- majusculus (Reut.) N: Espoo \& Järvenpää, Sa: Lappenranta. Rintala \& Rinne 2010:176.

- vicinus (Rib.) Ab: Turku, N: Helsinki. ibid.:176.

- laevigatus (Fieb.). Finland. Albrecht et al. 2008:7.

Coranus aethiops Jak. Ab, N, St, Ta, Sa, Oa, Kb. Rintala \& Rinne 2010:185.

Aradus montandoni Reut. Kb: Ilomantsi. ibid.:195.

Nysius groenlandicus (Zett.) (sp.dist.) Lk, Le, Li. ibid.:199.

Geocoris grylloides (L.) Ab: Dragsfjärd (P. Ahlroth). Albrecht et al. 2006:5, Rintala \& Rinne 2010:203.

[Oxycarenus lavaterae (F.)] Ka: Hamina (S. Karjalainen). Albrecht et al. 2006:5, Rintala \& Rinne 2010:268.

Tropidophlebia costalis (Herr.-Sch.) Sa: Valkeala. Rintala \& Rinne 2010:207.

Graptopeltus lynceus (F.) Al: Geta \& Lemland. Albrecht et al. 2006:5, Rintala \& Rinne 2010:219.

Pyrrhocoris apterus (L.) Sa: Imatra. Jantunen 2010 Suomen Luonto 69(8):13.

Bathysolen nubilus (Fall.) Ab, N, Sa. Rintala \& Rinne 2010:240.

Elasmostethus minor Horv. Al, Ab. ibid.:249.

Aelia rostrata Boh. Kl: Parikkala. ibid.:258.

Graphosoma lineatum (L.) Ab, N. Hem1.

Kelisia confusa Linnav. Al: Jomala. Albrecht et al. 2006:8.

Anakelisia perspicillata (Boh.) Al: Sund. ibid.:8.

Eurybregma porcus (Emel.) Kb: Tohmajärvi (M. Pajari). ibid.:8.

Ditropis flavipes (Sign.) Ka: Hamina. Hem2.

Delphacodes capnodes (Scott) St: Yläne, Ta: Kuru. Albrecht et al. 2006:8.

Acanthodelphax spinosa (Fieb.) Ab, N, Ta, Sa . ibid.:8; Söderman 2007 The Finnish Environment 7:22.

Florodelphax leptosoma (Flor) N: Helsinki. Hem2.

Xanthodelphax xantha Vilb. Sa, Kl. Albrecht et al. 2006:8. 
Ribautodelphax vinealis Biem. Ab, Ta, Ob, Ok, Lk, Le, Li. ibid.:8.

Ommatidiotus inconspicuus Stål N: Hanko. ibid.:8.

Aphrophora salicina (Gze.) Ab, N. Hem2.

Neophilaenus pallidus Haupt Al. ibid.

Macropsis albae Wagn. Finland. Albrecht et al. 2008:12.

- flavida Vilb. $\mathrm{O}$, Ob. Hem2.

- gravensteini Wagn. Ab: Pojo, $N$ : Elimäki. ibid.

- notata (Proh.) $A b$ : Karis. ibid.

- ocellata Prov. $N$ : Borgå. ibid.

- remanei Nick. St, Ta, Kb, Ob. ibid.

Anaceratagallia lithuanica Vilb. N, Sa. Albrecht et al. 2006:9; Söderman 2007:30.

Acericerus heydenii (Kirschb.) Ab: Nauvo \& Turku. Albrecht et al. 2006:9.

Metidiocerus crassipes (J.Sahlb.) Ob: Rovaniemi. Hem2.

Tremulicerus distinguendus (Kirschb.) Al, Ab, Sa. Söderman 2007:31.

Aphrodes diminuta Rib. N, St, Ta, Kb, Om, Lk. Albrecht et al. 2006:9.

Kybos volgensis (Vilb.) N: Ingå (G. Söderman). ibid.:9.

- rufescens (Mel.) N: Helsinki. Hem2.

- mucronatus (Rib.) Al. ibid.

Edwardsiana lethierryi (Edw.) Al: Lemland. Albrecht et al. 2006:9.

- plurispinosa (Wagn.) Al, Ab, N. ibid.:9.

- ulmiphaga Wils. \& Cl. (hippocastani auct.) Al, Ab, N. ibid.:9.

Linnavuoriana intercedens (Linnav.) (sp. dist.) $A l, A b$, $N, \mathrm{Ta}, \mathrm{Sa}, \mathrm{Kb}, \mathrm{Ok}$. ibid.:9.

Eupteryx stachydearum (Hardy) Al: Lemland \& Finström. ibid.: 10.

- aurata (L.) N: Elimäki. Hem2.

- decemnotata Rey $N$ : Helsinki. ibid.

Zygina schneideri (Günth.) Ab: Turku. ibid.

Circulifer haematoceps (Muls.\& R.) Om: Lohtaja (G. Söderman). Albrecht et al. 2006:10.

Macrosteles oshanini Razv. Ab: Nauvo. ibid.:10.

- sardus Rib. Ab, N. Hem2.

Doratura exilis (Horv.) N: Borgå. ibid.

Cicadula nigricornis (J.Sahlb.) Ab, N. Albrecht et al. 2006:10.

- rubroflava Linnav. Ab-Ks. Hem2.

Fieberiella septentrionalis Wagn. $N$ : Hangö. ibid.

Perotettix orientalis (Anufr.) Ta: Ruovesi. Albrecht et al. 2006:10.

- pictus (Leth.) Ta: Valkeakoski (J. Kirjavainen). ibid.: 10 .

Metalimnus obtusus Emel. $N$, Sa, Kb. Söderman 2007:55.

Psammotettix lapponicus Oss. Le: Kilpisjärvi (K. Mattila). Albrecht et al. 2006:11.
- slovacus Dlab. St, Ta, Sa, Kl, Oa. ibid.:11; Söderman 2007:57.

Adarrus multinotatus (Boh.) Ta: Valkeakoski (J. Kirjavainen). Albrecht et al. 2006:11.

Pinumius areatus Rib. Kl: Rautjärvi (M. Koponen). ibid.:11.

Cosmotettix evanescens Oss. Ab, Ta, Sa. ibid.:11; Söderman 2007:59.

Craspedolepta campestrella Oss. $\mathrm{Ka}$, Ta, Sa. Albrecht et al. 2008:17. Hem3.

Psylla buxi(L.) N, Ta. Albrecht et al. 2008:17. Hem3.

Cacopsylla pyricola (Schrank) Ka, Ta. Albrecht et al. 2006:11.

- pulchra (Zett.) Al-Kl, Kb, Ok. Albrecht et al. 2008:18. Hem3.

Bactericera parastriola Conc. et al. Li: Inari. Albrecht et al. 2008:18. Hem3.

- substriola Oss. Ok: Kuhmo. Albrecht et al. 2008:18. Hem3.

Trioza cirsii Löw Ab, N, Ta. Albrecht et al. 2008:18. Hem3.

- tatrensis Klim. Li: Utsjoki. Albrecht et al. 2008:18. Hem3.

Adelges lapponicus (Chol.) Li: Utsjoki. Albrecht et al. 2008:18. Hem4.

- tardus (Dreyf.) Ab-Lk. Albrecht et al. 2008:18. Hem4.

Acanthochermes quercus Koll. Ab: Turku Ruissalo. Albrecht et al. 2006:12.

Cinara cupressi (Buckt.) Ab, N, Ka. Albrecht et al. 2008:25. Hem4.

Trama rara Mordv. $N$ : Sjundeå. Albrecht et al. 2008:25. Hem4.

Anoecia nemoralis Börn. Ab, N, Ta, Sa. Albrecht et al. 2008:19. Hem4.

Pachypappa warshavensis (Nason.) Ka: Virolahti. Albrecht et al. 2008:19. Hem4.

Pemphigus passeki Börn. Ab, N, Sa. Albrecht et al. 2008:19. Hem4.

- immunis Buckt. Finland. Albrecht et al. 2008:19.

Subsaltusaphis lambersi (Quedn.) Ab, N, Sa. Albrecht et al. 2008:20. Hem4.

Sipha littoralis (Walk.) Ab, Sa, Ob. Albrecht et al. 2008:20. Hem4.

Aphis loti Kalt. $N$ : Helsinki (A. Albrecht). Albrecht et al. 2006:14.

- ruborum (Börn.) N: Sibbo (A. Albrecht). ibid.:14.

- etiolata Stroy. Sa: Rautjärvi. Albrecht et al. 2008:21. Hem4.

- nepetae Kalt. Ab: Turku. Albrecht et al. 2008:21. Hem4.

- rumicivora Heie Al-Kb. Albrecht et al. 2008:21. Hem4.

Schizaphis phlei Orlob N. Albrecht et al. 2008:21. Hem4. 
Dysaphis aizenbergi (Shap.) Al: Eckerö (A. Albrecht). Albrecht et al. 2006:14.

- ossiannilssoni Stroy. Ab: Dragsfjärd. Albrecht et al. 2008:22. Hem4.

- maritima (HRL) N. Albrecht et al. 2008:22. Hem4.

Ceruraphis eriophori (Walk.) Ab, N, Ta, Sa. Albrecht et al. 2008:22. Hem4.

Cavariella cicutae (Koch) Ta: Janakkala. Albrecht et al. 2008:22. Hem4.

Myzus nicotianae Blackm. N: Helsinki. Albrecht et al. 2008:23. Hem4.

Macrosiphum lisae Heie Om: Pyhäjoki. Albrecht et al. 2006:14.

Uroleucon rapunculoides (Börn.) Ab: Turku. ibid.:15.

- hypochoeridis (F.) Sa, Kb. Albrecht et al. 2008:24. Hem4.

- leontodontis (HRL) Ab-Ob. Albrecht et al. 2008:24. Hem4.

Amphorophora rubi (Kalt.) Ab-Lk. Albrecht et al. 2008:24. Hem4.

Atrococcus paludinus (Gr.) $N$ : Hyvinkää, $K b$ : Ilomantsi. Albrecht et al. 2006:15.

Peliococcus calluneti (Lid.) Kb: Ilomantsi. Albrecht et al. 2008:25. Hem3.

Trionymus perrisii (Sign.) Finland. Albrecht et al. 2008:25.

[Rhizoecus cactitans (Hambl.)] Finland. ibid.:25.

Acanthococcus uvaeursi (L.) Ab: Vihti. Hem3.

Pseudochermes fraxini (Kalt.) Finland. Albrecht et al. 2008:25.

Eulecanium tiliae (L.) Ab: Karis. Albrecht et al. 2008:26. Hem3.

Leucaspis pini (Hart.) Ab, N. Albrecht et al. 2008:26. Hem3.

- lowi Colv. [Lepidosaphes loewi] N: Helsinki (G. Söderman). Albrecht et al. 2006:15.

Present number: $1,428-7+128=1,549$ spp.

\section{Thysanoptera}

Kettunen, J. 2008. Suomen ripsiäisten luettelo / Check-list of Finnish Thysanoptera. http://www.sci.utu.fi/projects/biologia/elainmuseo/hemi/tyoryhma/Checklist_of_Finnish_Thysanoptera.pdf (2.6.2008)

Additions

Aeolothrips melaleucus Halid. Finland. Kettunen 2008: 1 .

Dendrothrips degeeri Uzel Finland. ibid.:1.

Neohydatothrips abnormis (Karny) Finland. ibid.:1. [Echinothrips americanus Morg.] Finland. ibid.:1. [Frankliniella occidentalis (Perg.)] Finland. ibid.:1. Thrips praetermissus Priesn. Finland. ibid.:2. [ - palmi Karny] Finland. ibid.:2.
Chirothrips pallidicornis Priesn. Finland. ibid.:2.

Taeniothrips inconsequens (Uzel) Finland. ibid.:2 .

Aptinothrips elegans Priesn. Finland. ibid.:2.

Haplothrips acanthoscelis (Karny) Finland. ibid.:2.

- kurdjumovi Karny Finland. ibid.:2.

- propinquus Bagn. Finland. ibid.:2.

- setigeriformis Fáb. Finland. ibid.:2.

- tritici (Kurdj.) Finland. ibid.:2.

Phlaeothrips bispinosus Priesn. Finland. ibid.:2.

Hoplandrothrips bidens (Bagn.) Finland. ibid.:3.

Hindsiothrips oettingeni (Priesn.) Finland. ibid.:3.

Hoplothrips fungi (Zett.) Finland. ibid.:3.

- grisescens (Priesn.) Finland. ibid.:3.

- pallicornis Crawf. Finland. ibid.:3.

- polysticti (Moris.) Finland. ibid.:3.

- unicolor (Vuill.) Finland. ibid.:3.

Maderothrips longisetis (Bagn.) Finland. ibid.:3.

Present number: $118+21=139$ spp.

\section{Neuroptera}

Ahlroth, P. 2010. Suomen verkkosiipiset ja kärsäkorennot / The Neuroptera s.1. and Mecoptera of Finland. http://www.sci.utu.fi/projects/biologia/elainmuseo/hemi/neur/verkmaps.htm (10.8.2010)

Additions

Hemerobius gilvus Stein N: Hangö. Ahlroth 2010.

Micromus variegatus (L.) Al-Ka. ibid.

Chrysopa walkeri McL. Sa: Joutseno. ibid.

Present number: $63+3=66$ spp.

\section{Coleoptera}

Albrecht, A., Karjalainen, S., Salokannel, J. \& The Finnish expert Group on Coleoptera. 2010. Suomen kuoriaisatlas/ Atlas över Finlands skalbaggar/ Atlas of the beetles of Finland. http://www.fmnh.helsinki.fi/elainmuseo/kovakuoriaiset (15.3.2010)

Deletions

Choleva agilis (Ill.) (f.d., =C. angustata (F.)). Ruzicka \& Vávra 2003 Mem. Entomol., Internat. 17:158.

Onthophagus similis (Scr.) (f.d., $=O$. fracticornis (Preyssl.). Roslin \&Heliövaara 2007 Suomen lantakuoriaiset, p. 180.

Lamprobyrrhulus nitidus (Schall.) Recorded outside Finland. Silfverberg 2008 [2009] Sahlbergia 14:55.

Atomaria pseudaffinis Johns.\& Str. (= A. affinis (F.Sahlb:)). Johnson 2006 Entomol. Gaz. 57:55.

Cryptocephalus flavipes F. (f.d., $=C$. bameuli Duhald.). Silfverberg 2008 [2009] Sahlbergia 14:54. 
Additions

Graphoderus austriacus (Sturm) Al: Eckerö (T. Clayhills). Albrecht et al. 2010.

!Cybister lateralimarginalis (DeG.) Ab: Dragsfjärd Örö. Kullberg 2007 Suomen Luonto 66(6):12. Hildén et al. 2007 Memoranda Soc. Fauna Flora Fenn. 83:85.

Bembidion obtusum Serv. Al: Eckerö (E. Helve). Hildén et al. 2010 Memoranda Soc. Fauna Flora Fenn. 86:87.

Anisodactylus signatus (Panz.) Sa: Juva. Martikainen 2006 Sahlbergia 11:7.

Bradycellus harpalinus (Serv.) N: Hanko (P. Rassi). Albrecht et al. 2010.

Masoreus wetterhallii (Gyll.) Ab: Hitis. ibid.

Helophorus pumilio Er. Ka: Virolahti (S. Karjalainen). ibid.

!Berosus fulvus Kuw. Al, Ab. Valtonen 2010 Sahlbergia 16:1.

Acrotrichis nana Strand $\mathrm{Sa}$ : Pertunmaa. Martikainen 2006:8.

- suecica Sundt N: Espoo (P. Rassi). Albrecht et al. 2010.

Nevraphes perssoni Franz Ob: Pudasjärvi (P. Rassi). ibid.

Phyllodrepa vilis (Er.) Al. ibid.

Omalium oxyacanthae Grav. N: Espoo (P. Rassi). ibid.

Anthobium unicolor (Marsh.) Al: Föglö (M. Pentinsaari). ibid.

Megarthrus hemipterus (Ill.) Sb: Savonranta. ibid.

!*Euplectus lapponicus Löbl. \& Matt. Li: Inari. Löbl \& Mattila 2010 EF 21:182.

Batrisodes buqueti (Aubé) Sb: Savonranta. Albrecht et al. 2010.

Aleochara signata (J.Sahlb.) (sp.dist.) Ab: Åbo. Löbl \& Smetana 2004 Catal. Pal. Col. 2:360; Assing 2009 Beitr. Entomol. 59:64.

Dexiogyia forticornis (Strand) Ta: Heinola (J. Mattila). Albrecht et al. 2010.

Acrotona pilosicollis (Brund.) Sa: Ruokolahti. ibid.

Agaricochara latissima (Steph.) Al: Kökar (P. Rassi). ibid.

Tachyusida gracilis (Er.) Ta: Loppi. ibid.

Oligota granaria Er. $A b$ : Dragsfjärd. ibid.

- pumilio Kiesw. Al, N, Ta, Sa. ibid.

Stenus kiesenwetteri Rosh. Ab: Hitis. ibid.

Gabrius piliger Muls. \& R. Al: Lemland Nåtö. Clayhills 2010 Memoranda Soc. Fauna Flora Fenn. 85:97.

Quedius persimilis Muls. \& R. Al: Eckerö (T. Clayhills). Albrecht et al. 2010.

Semijulistus callosus (Sols.) Om: Pyhäntä (S. Kaartinen), $O b$ : Pudasjärvi (J. Mattila). ibid. \& Mattila pers.comm.

Epuraea melanocephala (Marsh.) Al. ibid.
Meligethes matronalis Aud. \& Sp. Al, Ab, N, Tb. ibid.

Cryptophagus intermedius Bruce Al: Lemland Nåtö. Clayhills 2010:97.

Aridius bifasciatus (Rtt.) N: Espoo (P. Rassi). Albrecht et al. 2010.

Mordellistena secreta Hor. Sa: Taipalsaari (I. Rutanen). ibid.

Uloma culinaris (L.) Kb: Kitee (P. Turunen). ibid.

Oedemera subrobusta Nak. Sa: Joutseno. Valtonen et al. 2006 Sahlbergia 11(2):38. Höjer 2008 Entomo1. Tidskr. 129:93.

Pseudovadonia livida (F.) Sa, Kl. Albrecht et al. 2010.

Cryptocephalus bameuli Duhald. (flavipes auct.) Ab: Eura \& Lojo. Silfverberg 2008 [2009] Sahlbergia 14:54.

Longitarsus plantagomaritimus Dollm. Ka: Hamina (S. Karjalainen). Albrecht et al. 2010.

Psylliodes chalcomera (Ill.) Kl: Simpele. ibid.

Apion frumentarium (L.) $\mathrm{N}, \mathrm{Ka}$. ibid.

! - gnarum Faust Ta: Lahti (J. Salokannel). Mattila 2008 Diamina 17:38.

[Otiorhynchus armadillo (Rossi)] $N$ : Järvenpää. Albrecht et al. 2010

[Otiorhynchus tenebricosus Hbst. (lugdunensis Boh.)] $N$ : Järvenpää. ibid.

Polydrusus formosus (Mayer) Al: Mariehamn, $N$ : Espoo (E. Helve). Hildén et al. 2010:87. Albrecht et al. 2010.

Hypera dauci (Ol.) Ka: Vehkalahti. Albrecht et al. 2010.

Anthonomus pomorum (L.) N, Ka, Sa. ibid.

Pelenomus quadricorniger (Colon.) Kl: Parikkala. ibid.

Ceutorhynchus pectoralis Wse. Kl: Simpele \& Parikkala. ibid.

Ceutorhynchus melanostictus (Marsh.) Al. ibid.

Hylastes attenuatus Er. Ab: Iniö. ibid.

Present number: $3,657-5+48=3,700$ spp.

\section{Trichoptera}

Additions

Ithytrichia clavata Mort. Ab: Karjaa. Rinne \& Salokannel 2008 Diamina 17:2.

Present number: $215+1=216$ spp.

\section{Lepidoptera}

Deletions

Caloptilia semifascia (Haw.) (f.d., $=C$. jurateae Bengtss.). Bengtsson 2010 Entomol. Tidskr. 131:107.

Oxyptilus distans (Zell.) (f.d., = O. tristis (Zell.)). Välimäki et al. 2009 Baptria 34:50. 
Additions

Bucculatrix noltei Petry Ka: Virolahti (J.P. \& P. Kaitila). Mutanen et al. 2008b Baptria 33(1):8.

Bucculatrix chrysanthemella Reb. Sa: Kangasniemi. Siloaho 2008 Baptria 33:142. - import?

*Caloptilia jurateae Bengtss. (semifascia auct.) Finland. Bengtsson 2010 Entomol. Tidskr. 131:107.

Phyllonorycter trifoliellus (Geras.) $N$ : Hanko, Ka: Virolahti. Saarela 2009 Baptria 34:39. Välimäki \& Kaitila 2010 Baptria 35:86.

- comparellus (Dup.) N: Vantaa (J.-P. Kaitila). Välimäki \& Kaitila 2010:79.

Parornix fagivora (Frey) St: Rauma (O. Helminen). Sippola 2009 Baptria 34:39.

Cameraria ohridella Deschk. \& Dim. N: Hanko \& Espoo. Kaitila 2008 Baptria 33:119. Välimäki \& Kaitila 2010:86.

Agonopterix yeatiana (F.) Ka: Virolahti. Kaitila 2008:119.

*Elachista deriventa Kaila \& Mut. Ta, Sa, Kl, Kb. Kaila et al. 2008 EF 19:185. Välimäki \& Kaitila 2010:82.

- baltica Her. Lk: Sodankylä \& Pelkosenniemi. Mutanen et al. 2008b:9.

* - saarelai Kaila \& Sipp. Ta: Tampere. Kaila \& Sippola 2010 EF 21:131.

Coleophora filaginella Fuchs N: Porvoo (P. Hirvonen), Ta: Valkeakoski (L. Sippola). Saarela \& Sippola 2006 Baptria 31:40. Mutanen et al. 2008a Baptria 32:41. Välimäki \& Kaitila 2010:83.

Monochroa simplicella (Lien. \& Zell.) $N$ : Ruotsinpyhtää. Nupponen 2008 Baptria 33:120.

Scrobipalpa proclivella (Fuchs) $N$ : Hanko \& Helsinki. Laasonen \& Laasonen 2008 Baptria 33:120. Välimäki \& Kaitila 2010:86.

Dichomeris rasilella (H.-S.) Ka: Virolahti. Kaitila 2008 Baptria 33:121. Välimäki \& Kaitila 2010:86.

- derasella (D. \& Sch.) N: Sipoo (O. Helminen, L. Jalonen \& I. Seuranen). Välimäki \& Kaitila 2010:80

Synanthedon soffneri Špat. Ta: Pälkäne. Martikainen 2010 Diamina 19:18. Välimäki \& Kaitila 2010:80.

Acleris effractana (Hübn.) Ab, N, Ka, Ta, Kl, Oa. Mutanen et al. 2008b:9.

- cristana (D. \& Sch.) Al: Lemland, Ab: Korpo. Mutanen 2008 Baptria 33:121. Välimäki \& Kaitila 2010:86.

Cochylidia moguntiana (Rössl.) N: Tammisaari (L. Kaila). Mutanen et al. 2008b:10.

Eana derivana (La H.) Al: Kökar. ibid.:10.

Cnephasia genitalana P. \& Metc. Ab: Korpo \& Dragsfjärd. ibid.:10.

Archips crataeganus (Hübn.) Ab: Dragsfjärd (E. \& L. Laasonen \& R. Martikainen). ibid.:11.
Stenoptilia nolckeni (Tengstr.) Sa: Kangasniemi (R. Siloaho). Kullberg 2008b Baptria 33:122.

Oxyptilus tristis (Zell.) Ab, N. Mutanen 2008:122. Välimäki \& Kaitila 2010:86.

Amblyptilia acanthadactyla (Hübn.) $A l, A b, N$. Mutanen 2009 Baptria 34:40. Välimäki \& Kaitila 2010:88.

Porrittia galactodactyla (D. \& Sch.) Ab: Salo, $N$ : Espoo \& Helsinki. Mutanen 2008:125. Välimäki \& Kaitila 2010:88.

Agriphila deliella (Hübn.) Ka: Virolahti (H. Luoma). Mutanen et al. 2008b:12.

Thisanotia chrysonuchella (Scop.) Ab: Dragsfjärd (A. \& J. Kullberg). ibid.:11.

Epascestria pustulalis (Hübn.) Ab: Dragsfjärd (A. \& J. Kullberg). ibid.:12.

Anania verbascalis (D. \& Sch.) Al, N, Ka. Mutanen et al. 2008b:12. Välimäki et al. 2008b Baptria 33(3): 17

Euchloe ausonia (Hb.) N: Helsinki (I. Jalas). Kolev, Kullberg \& Paukkunen 2010 Baptria 35:42.

Lycaena tityrus (Poda) Ka: Hamina (T. Nupponen) Välimäki, Kaitila \& Lehto 2010 Baptria 35:44.

Erebia euryale euryaloides Tengstr. Kb: Lieksa (P. Grotenfelt). Kullberg 2007 Suomen Luonto 66(2):14. Hildén et al. 2007 Memoranda Soc. Fauna Flora Fenn. 83:85. Kullberg 2008a Baptria 33:47-48.

Scopula nigropunctata (Hufn.) Ab: Houtskär Jungfruskär (J.-P. Kaitila \& M. Rantala). Mutanen et al. 2007 Baptria 32:41.

Scopula marginepunctata (Gze.) N: Hangö (K.-E. Lundsten). Välimäki 2008 Baptria 33(2):48. Välimäki et al. 2008a Baptria 33(2):70.

Asthena anseraria (H-S) N. Välimäki 2008:48-49.

Stegania cararia (Hübn.) Ka: Virolahti. Kaitila \& Jalonen 2008 Baptria 33:49. Välimäki et al. 2008a:70.

Parectropis similaria (Hufn.) Ab, Ka. Kaitila, Koski \& Öhman 2008 Baptria 33:49-50. Välimäki et al. Baptria 33:68.

Pericallia matronula (L.) Ab: Dragsfjärd (T. Klemetti). ibid.:42.

Dysgonia algira (L.) N. Välimäki 2008 Baptria 33(2):50. Välimäki et al. 2008a:74.

Eublemma parvum (Hübn.) Ab, Ka. Kaitila 2008 Baptria 33(2):50. Välimäki et al. 2008a:74.

Cucullia praecana Ev. Ab, Ka. Välimäki 2008:50-51.

- tanaceti (D. \& Sch.) N: Hanko. Koski 2010 Baptria 35:45.

Apamea epomidion (Haw.) N: Tammisaari Jussarö (T. \& K. Nupponen). ibid.:43.

Hadena filograna (Esp.) N, Ka. Välimäki 2008:51. Välimäki et al. 2008a:69.

Mythimna loreyi (Dup.) N: Kirkkonummi (D. 
Lindblom). Välimäki 2008:51. Välimäki et al. 2008a:71.

Epilecta linogrisea (D. \& Sch.) Ab: Dragsfjärd (J. Jokinen \& R. Teriaho). Välimäki 2008:52. Välimäki et al. 2008a:70.

Xestia ditrapezium (D. \& Sch.) N. Välimäki 2008:52.

Dichagyris signifera (D. \& Sch.) N: Tammisaari Jussarö (T. \& K. Nupponen). Nupponen 2007 Baptria 32:42.

Agrotis bigramma (Esp.) Ab: Dragsfjärd (J. \& A. Kullberg). Kullberg 2008a:52-53. Välimäki et al. 2008a:71.

Present number: $2,509-2+51=2,558$ spp.

\section{Diptera}

Haarto, A. \& Kerppola, S. 2007. Suomen kukkakärpäset. Helsinki, 647 pp.

Winqvist, T. \& Kahanpää, J. 2007. Checklist of Finnish flies: superfamilies Tephritoidea and Sciomyzoidea. Sahlbergia 12:20-32.

Paasivirta, L. 2009. Chironomidae (Diptera: Nematocera) in the biogeographical provines of Finland. http:// www.ymparisto.fi/download.asp? contentid $=82649$ (20.3.2009)

Deletions

Pseudosmittia brevitarsis Brund. (= P. ruttneri Strz. \& Thien.). Sæther \& Ferrington 2003 Zootaxa 322:4.

Micropsectra bidentata (Goethgh.) (f.d., $=M$. pallidula (Meig.)). Stur \& Ekrem 2006 Zool. J. Linn. Soc. 146:201.

Zavrelia atrofasciata Kieff. (= Z. pentatoma Kieff. \& B.). Ekrem \& Stur 2009 Europ. J. Ent. 106:132.

Keroplatus sesioides (Wahlb.) (=K. tipuloides Bosc) Kjærandsen et al. 2007 Ins. Syst. Evol. Suppl. 65:20.

Mycomya kingi Edw. (= M. shermani Garr.). ibid.:31.

Brevicornu boreale (Lundstr.) (= B. griseolum (Zett.)). ibid.:61

Cordyla sixi (Barendr.) (= C. pusilla Edw.). ibid.:63.

- bergensis (Barendr.) (=C. nitidula Edw.). Jakovlev et al. 2006 Sahlbergia 11:23.

Pseudorymosia optiva (Dziedz.) (f.d., = P. fovea (Dziedz.)). ibid.:32.

Dynatosoma norwegiense Zaitz. \& Økl. (= D. thoracicum (Zett.)). Kjærandsen et al. 2007:78.

Mycetophila assimilis Mat. (= M. signatoides Dziedz.). ibid.:85.

Syntormon denticulatus (Zett.) (f.d., = S. freymuthae Loew). Kahanpää 2006 Dipterol. Res. 17:124.

Bradysia cellarum Frey (= B. tilicola $($ Loew) (amoena (Winn.))). Menzel \& Heller 2006 Stud. Dipterol. 13:213.

- pratincola Tuomik. (= B. affinis (Zett.)). Heller et al. 2009 Sahlbergia 15:25.
Dolichopus consimilis Wahlb. (= D. picipes Meig.). Kahanpää 2008 Zootaxa 1689:51.

- cruralis Wahlb. (=D. lepidus Staeg.). ibid.:51.

- parvicaudatus Zett. (=D. plumipes (Scop.)). ibid.:51. - pectinitarsis Stenh. (=D. plumipes (Scop.)). ibid.:51.

Eupeodes borealis (Dus. \& Las.) (f.d., =E. punctifer Frey). Haarto \& Kerppola 2008 Sahlbergia 13(2):39.

Parasyrphus relictus (Zett.) (f.d.). ibid.:39.

Paragus majoranae Rond. (f.d., = P. pecchiolii Rond.). ibid.:39.

Pipiza notata Meig. (spec. dub.). Haarto \& Kerppola 2007:452.

Cheilosia pallipes Loew (f.d., = C. flavissima Beck.). Haarto \& Kerppola 2007:615. Haarto \& Kerppola 2008:39.

Pipunculus spinipes Meig. (= P. campestris Latr.). Kehlmaier 2008 Zootaxa 1672:6.

Claraeola halterata (Meig.) (f.d.). Kehlmaeir \& Ståhls 2008 Sahlbergia 13(2):66.

Clistoabdominalis trochanteratus (Beck.) (f.d., = Eudorylas fuscipes (Zett.)). ibid.:66.

Dasydorylas roseri (Beck.) (f.d., = Eudorylas fuscipes (Zett.)). ibid.:66.

Eudorylas pannonicus (Beck.) (f.d., = E. restrictus Coe). ibid.:66.

Thecophora atra (F.) (f.d.). Kahanpää 2007 EF18:172. - melanopa Rond.(f.d., =T. distincta Wied.). ibid.:170. Melieria obscuripes (Loew) (f.d., = M. omissa (Meig.)). Winqvist \& Kahanpää 2007:25.

Campiglossa irrorata (Fall.) Recorded outside Finland. Söderman et al. 2007 Sahlbergia 12:10.

- tesselata (Loew) (f.d., = C. difficilis (Hend.)). ibid.: 10 .

Dioxyna sororcula (Wied.) Dubious record. ibid.:11.

Tephritis dioscurea (Loew) (f.d., = T. rydeni (Her.)). ibid.: 7 .

- nigricauda (Loew) Dubious record. ibid.:7.

Orygma luctuosum Meig. Recorded outside Finland. Winqvist \& Kahanpää 2007:26.

Prochyliza lundbecki (Duda) (f.d., = P. varipes (Meig.)). ibid.:24.

Allopiophila dudai (Frey). Recorded outside Finland. ibid.:24.

Chaetolonchaea dasyops (Meig.) (f.d., $=C$. pallipennis (Zett.)). ibid.:22.

Lonchaea alni Ringd. (= L. corusca Czerny). ibid.:28. - britteni Coll. (= L. corusca Czerny). ibid.:28.

- bruggeri Morge Dubious record. ibid.:22.

- contigua Coll. Dubious record. ibid.:23.

- difficilis Hackm. Dubious record. ibid.:23.

- flavidipennis Zett. (f.d., = L. limatula Coll.) ibid.:28.

- hirticeps Zett. (f.d.). ibid.:23.

- sarekensis Frey (=L. deutschi Zett.) ibid.:28. 
Species reported and later deleted

Keroplatus tuvensis Zaitz. Ok: Kuhmo. Polevoi et al. 2006 EF 17:164; = K. testaceus (Dahlm.) Kjærandsen et al. 2007 Ins. Syst. Evol. Suppl. 65:20.

Rhaphium auctum Loew Al: Jomala (R. Storå); Om: Jakobstad (R. Frey). Kahanpää \& Grichanov 2006 EF 17:76; f.d., = R. monotrichum Loew, Kahanpää 2006 Dipterol. Res. 17:123.

Syntormon aulicus (Meig.) Ab: Karislojo (W. Hellén); $N$ : Esbo (W. Hellén). Kahanpää \& Grichanov 2006:76; f.d., = S. tarsatus Meig., Kahanpää 2006:123.

Dolichopus albifrons Loew Ab: Korpo Jurmo (M. Meinander). Kahanpää \& Grichanov 2006 EF 17:75; f.d., = D. caligatus Wahlb., Kahanpää 2006:123.

Medetera micacea Loew N: Helsingfors (W. Hellén); Ta: Valkeala (R. Frey). Kahanpää \& Grichanov 2006:76; f.d., Kahanpää 2006:123.

Additions

Dolichopeza nitida Mik Lk: Sodankylä, Li: Utsjoki. Salmela 2008 w-album 6:12, 29.

Prionocera abscondita Lacksch. Le: Malla, Li: Utsjoki. ibid.:12, 29.

- woodorum Brodo Li: Utsjoki. ibid.:12

Tipula laetibasis Alex. Tb: Petäjävesi (K. Kulmala), $L k$ : Kolari (J. Jakovlev \& J. Penttinen). Salmela et al. 2007a Sahlbergia 12:33.

- octomaculata Savch. Ks: Taivalkoski. Salmela \& Autio 2007b Zootaxa 1527:53.

- invenusta Ried. Lk, Le, Li. Salmela 2008:12.

- luna Westh. Al: Jomala. Autio \& Salmela 2010 Memoranda Soc. Fauna Flora Fenn. 86:49.

- jutlandica Niels. Kl: Parikkala (J. Mattila). Salmela 2009 Zoosymposia 3:254.

- pabulina Meig. N. ibid.:253.

- stenostyla Savch. Ta, Sb. ibid.:257.

- chonsaniana Alex. (sp. dist.) Oa, Ob, Ks. Salmela \& Autio 2009 EF 20:52.

Cylindrotoma borealis Peus (sp.dist.) Ab, Ta, Oa, Tb. Salmela \& Autio 2007a Dipterol. Res. 18:49.

Paradelphomyia nigrina (Lacksch.) Le: Enontekiö. Salmela 2008:10.

Neolimnomyia batava (Edw.) Ab: Karkkila, Ta: Tampere. Salmela et al. 2007b Memoranda Soc. Fauna Flora Fenn. 83:45. Salmela 2010 Diamina 19:12.

Symplecta chosenensis (Alex.) $N$ : Mäntsälä. Kahanpää \& Salmela 2007 Sahlbergia 12:45.

Molophilus pullus Lacksch. N: Nurmijärvi. ibid.:39, 45.

Ormosia loxia Starý N: Nurmijärvi. ibid.:40, 45.
Tasiocera exigua Savch. Ab, N, Ta. Salmela et al. 2007b:45.

Gnophomyia viridipennis (Gimm.) Al: [Lemland \& Föglö]. Hancock 2008 Sahlbergia 14:14.

Helius longirostris (Meig.) Ta: Ruovesi. Salmela et al. 2007b:46.

Dicranomyia aperta Wahlgr. Lk: Kittilä. Salmela 2008:10.

- moniliformis Doane Lk: Kittilä. ibid.:10, 29.

- omissinervis Meij. Lk: Kittilä. ibid.:10.

- lulensis (Tjed.) Le: Enontekiö. ibid.:10.

* Metalimnobia charlesi Salm. \& St. Ta, Sa,Tb, Sb. Salmela \& Starý 2009 EF19:269. Salmela 2010:12.

- tenua Savch. \& Kriv. Ta: Ruovesi. Salmela et al. 2007b:46.

Tinearia lativentris (Berd.) Ab: Kiikala, Ta: Tampere. Salmela et al. 2007b.:39, 47. Salmela 2010:13.

Pneumia pilularia (Tonn.) Le: Saana, Li: Utsjoki. Salmela 2008:13.

Psychodocha itoco (Tog. \& Kom.) Ta: Ruovesi. Tb: Viitasaari. Salmela et al. 2007b:47. Salmela \& Autio 2007b:54.

Psychoda grisescens Tonn. Ta: Tampere, Li: Utsjoki. Salmela 2008:13. Salmela 2010:13.

Katamormia strobli Jez. Tb: Jyväskylä. Salmela \& Halme 2007 Diamina 16:6.

Peripsychoda auriculata (Curt.) Ab: Karkkila, $N$ : Nurmijärvi. Salmela et al. 2007b:47.

Trichopsychoda hirtella (Tonn.) Tb: Jyväskylä. Salmela \& Halme 2007:6.

Lepiseodina rothschildii (Eat.) Ta: Tampere. Salmela 2010:9, 13.

Dixella nigra (Staeg.) Ta: Loppi. Salmela et al. 2007b:39, 47.

- laeta (Loew) Lk, Le, Li. Salmela 2008:13.

Trichotanypus mariae Wirth \& Subl. Li. Paasivirta 2009:1.

Procladius crassinervis (Zett.) Al-Li. ibid.:2.

- fuscus Brund. Oa, Kb, Ob, Li. ibid.:2.

- suecicus Brund. Ta, Om, Li. ibid.:2.

- rufovittatus v.d.Wulp Al-Li. ibid.:2.

Natarsia nugax (Walk.) Ab. ibid.:2.

Conchapelopia aagaardi Murr. Ks, Le, Li. ibid.:1.

- hittmairorum Mich. \& Sp. Ab-Li. ibid.:1.

Hayesomyia tripunctata (Goetgh.) Ka, Ta, Sa, Kl. ibid.:1.

Krenopelopia nigropunctata (Staeg.) Ab-Ta. ibid.:1 .

Larsia atrocincta (Goetgh.) N-Lk. ibid.:1.

Pentaneurella katterjokki Fittk. \& Murr. Ob-Li. ibid.:2.

Rheopelopia maculipennis (Zett.) N-Li. ibid.:2.

Telopelopia fascigera Vern. $N$, Ka, Ta. ibid.:2.

Thienemannimyia laeta (Meig.) Ka, Tb. ibid.:2.

- vitellina (Kieff.) Oa,Ob. ibid.:2.

Xenopelopia falcigera (Kieff.) Ta. ibid.:2. 
Diamesa hyperborea Holmgr. Le, Li. ibid.:3.

- insignipes Kieff. Om-Li. ibid.:3.

Pseudodiamesa arctica (Mall.) Ta, Ob, Le, Li. ibid.:3.

Sympotthastia fulva (Joh.) Li. ibid.:3.

Telmatogeton japonicus Tokun. $N$ : Pyttis, Ka: Haapassari. Rautio, Paasivirta \& Brodin 2009 Aquatic Invas. 4:406.

Odontomesa fulva (Kieff.) Ab-Ok. Paasivirta 2009:3.

Aagardia protensa Saeth. Ab-Li. ibid.:3.

Abiskomyia paravirgo Goetgh. Tb-Li. ibid.:3.

Bryophaenocladius inconstans (Brund.) Ab-Li. ibid.:4.

- laticaudus Saeth. $A b, T b, L i$. ibid.:4.

- muscicola Kieff. $A b, N$. ibid.:4.

- nigrus Albu Ab. ibid.:4.

- subparallelus (Mall.) St, Lk, Li. ibid.:4.

- vernalis (Goetgh.) Sb. ibid.:4.

Cardiocladius fuscus Kieff. Ab-Sb ibid.:4.

Chaetocladius binotatus (Lundstr.) Le, Li. ibid.:4.

- dissipatus (Edw.) N-Le. ibid.:5.

- grandilobus Brund. Al-Li. ibid.:5.

- melaleucus (Meig.) N-Li. ibid.:5.

Corynoneura celtica Edw. Ka-Li. ibid.:5.

Cricotopus fuscus (Kieff.) Ab-Li. ibid.:5.

- pallidipes Edw. Ks-Li. ibid.:6.

- lygropis Edw. Tb, Ks. ibid.:6.

Dratnalia potamophylaxi (Fittk. \& Lell.) Ok. ibid.:6.

Eukiefferiella clypeata (Kieff.) Ab, N, Kl. ibid.:7.

- dittmari Lehm. Ab-Ks. ibid.:7.

- gracei (Edw.) St, Tb, Ob. ibid.:7.

- minor (Edw.) Ok-Li. ibid.:7.

Eurycnemus crassipes (Panz.) Ob. ibid.:7.

Heleniella serratosioi Ringe $A b-K s$. ibid.:7.

Heterotrissocladius brundini Saeth. \& Schn. Le, Li. ibid.:7.

Hydrobaenus fusistylus (Goetgh.) Ks, Le. ibid.:7.

- lapponicus Brund. Le. ibid.:7.

Krenosmittia boreoalpina (Goetgh.) Ab-Li. ibid.:7.

- camptophleps (Edw.) Le. ibid.:7.

Limnophyes aagaardi Saeth. Lk. ibid.:7.

- angelicae Saeth. Ob. ibid.:7.

- bidumus Saeth. Ob-Li. ibid.:7.

- brachytomus (Kieff.) Ok-Li. ibid.:8.

- edwardsi Saeth. Ab-Li. ibid.:8.

- margaretae Saeth. Le. ibid.:8.

- ninae Saeth. Ab-Li. ibid.:8.

- pentaplastus (Kieff.) Ab-Ks. ibid.:8.

- schnelli Saeth. Le. ibid.:8.

- spinigus Saeth. Ab-Li. ibid.:8.

- torulus Saeth. Le, Li. ibid.:8.

Metriocnemus acutus Saeth. Kb-Li. ibid.:8.

- beringiensis (Cranst. \& Ol.) Al-Li. ibid.:8.

- caudigus Saeth. Ab-Li. ibid.:8.

- eurynotus (Holmgr.) Ab-Li. ibid.:8.

- exilacies Saeth. Ab-Li. ibid.:8.
- intergerivus Saeth. Tb. ibid.:8.

Nanocladius parvulus (Kieff.) $N$-Li. ibid.:8.

Orthocladius fuscimanus Kieff. Ka, Le. ibid.:8.

- gelidorum (Kieff.) Ta-Li. ibid.:8.

- gelidus Kieff. Tb, Le, Li. ibid.:8.

- musester Saeth. Ab-Lk. ibid.:8.

- abiskoensis Thien. \& Kr. Ab. ibid.:8.

- ashei Sopon. Ka, Ta, Sa. ibid.:9.

- rivicola Kieff. Tb-Li. ibid.:9.

- rivulorum Kieff. Ta, Sa, Le, Li. ibid.:9.

- saxosus Tokun. Ks, $L k$, Li. ibid.:9.

- lapponicus Goetgh. Ob, Lk, Le, Li. ibid.:9.

- pedestris Kieff. Sa-Li. ibid.:9.

- rhyacobius Kieff. Ab-Li. ibid.:9.

- ruffoi Ross. \& Pr. Ab. ibid.:9.

- wetterensis Brund. St-Le. ibid.:9.

- holsatus Goetgh. Sa-Le. ibid.:9.

- schnelli Saeth. Ab-Li. ibid.:9.

- smolandicus Brund. Ab-Lk. ibid.:9.

Parachaetocladius abnobaeus (Wulk.) Ab-Kb. ibid.:9.

Paracricotopus niger (Kieff.) Ab, Ka, Om, Ok. ibid.:9.

Parakiefferiella finnmarkica Tuisk. Kb-Li. ibid.:9.

- subaterrima (Mall.) Ka, Oa, Ob, Lk. ibid.:10.

Paraphaenocladius exagitans (Joh.) Ab-Li. ibid.:10.

- intercedens Brund. Ta-Li. ibid.:10.

- pseudirritus Stren. Ab-Li. ibid.:10.

- triangulus Saeth. \& Wang Ob. ibid.:10.

Parasmittia carinata Stren. Al-Sb. ibid.:10.

Paratrissocladius excerptus (Walk.) Ab, Ok. ibid.:10.

Propsilocerus saetheri Wang, Liu \& Paas. Ab-Li. ibid.:10.

Psectrocladius conjungens (Brund.) Al-Li. ibid.:10.

- oligosetus Wulk. N-Li. ibid.:10.

- oxyura Langt. Al-Li. ibid.: 10.

- schlienzi Wulk. N-Li. ibid.:10.

Pseudorthocladius filiformis Kieff. Al-Li. ibid.:10.

Pseudosmittia albipennis (Goetgh.) Al, Ab. ibid.:10.

- angusta (Edw.) Ab-Li. ibid.:11.

- danconai (Marc.) N, Oa, Ok, Ob. ibid.:11.

- gracilis (Goetgh.) Ka-Li. ibid.:11.

- mathildae Albu Tb-Li. ibid.:11.

- obtusa Stren. Ab-Li. ibid.:11.

Rheocricotopus atripes Kieff. $A b-L k$. ibid.:11.

- glabricollis (Meig.) Kb. ibid.:11.

- reduncus Saeth. \& Schn. Tb-Li. ibid.:11.

Rheosmittia languida (Brund.) Ob, Li. ibid.:11.

Smittia edwardsi Goetgh. Al-Li. ibid.:11.

- foliacea (Kieff.) Al. ibid.:11.

- nudipennis (Goetgh.) Al-Li. ibid.:11.

- scutellosetosa Casp. Ab, Sa. ibid.:11.

- stercoraria Ross. Ab-Li. ibid.:11.

Stackelbergina praeclara Shil. \& Zel. Kb, Ks. ibid.:11.

*Tavastia yggdrasilia Brod. et al. N, Ka, St, Ta, Sa, Kl, $K b$. Brodin, Lundström \& Paasivirta 2008 Aquatic Ins. 30:262. 
Thienemannia gracilis Kieff. Ab-Ks. Paasivirta 2009:11.

Thienemanniella acuticornis (Kieff.) Ab-Li. ibid.:12.

- minuscula (Brund.) Al-Li. ibid.:12.

- obscura Brund. Ab, Ks, Lk, Li. ibid.:12.

Tokunagaia excellens (Brund.) Li. ibid.:12.

- scutellata (Brund.) Le, Li. ibid.:12.

- tonollii (Ross.) Ok-Li. ibid.:12.

Vivacricotopus ablusus Schn. \& Saeth. Ks, Le. ibid.:12.

Chironomus islandicus (Kieff.) Oa, Lk, Li. ibid.:13.

- salinarius Kieff. Ab. ibid.:13.

- carbonarius Meig. Ab, N, Ta. ibid.:13.

Cladopelma goetghebueri Spies Sb. ibid.:14.

Cryptochironomus denticulatus Goetgh. Ks. ibid.:14.

- obreptans (Walk.) Ab, Ta, Oa, Ob. ibid.:14.

Cryptotendipes darbyi (Subl.) Om, Ob. ibid.:14.

- pseudotener (Goetgh.) N. ibid.:14.

Demicryptochironomus neglectus Reiss Ka, Ta. ibid.:14.

Dicrotendipes notatus (Meig.) Ab, $N$, Ka. ibid.:14.

Glyptotendipes glaucus (Meig.) Ab-Oa. ibid.:14.

- viridis (Macq.) Ab, N, Ka. ibid.:14.

Harnischia fuscimana Kieff. $N$-Lk. ibid.:15.

Kiefferulus tendipediformis (Goetgh.) Al-Sb. ibid.:15.

Kloosia pusilla (L.) Ab, Kb. ibid.:15.

Lipiniella prima Shil. et al. Ta. ibid.:15.

Microtendipes rydalensis (Edw.) Ab-Kb. ibid.:15.

Parachironomus siljanensis Brund. Al-Li. ibid.:15.

- varus (Goetgh.) Al-Li. ibid.:15.

Paracladopelma undine (Town.) Ta, Ok, Ob, Ks, Le. ibid.:15.

Polypedilum acutum Kieff. Ab-Lk. ibid.:16.

- trigonus Town. Al-Lk. ibid.:16.

- aegyptium Kieff. Ks. ibid.:16.

- quadriguttatum Kieff. Ob, Lk. ibid.:16.

Sergentia baueri Wulk, et al. Ab-Ks. ibid.:16.

Stictochironomus maculipennis (Meig.) Ka, St, Sb, $O b, L k$. ibid.:16.

Cladotanytarsus cyrylae Giłka Kl. ibid.:17.

- gedanicus Giłka N, St, Oa, Ob, Li. ibid.:17.

- matthei Giłka Lk, Li. ibid.:17.

Micropsectra attenuata Reiss Ab-Kb. ibid.:17.

- calcifontis Stur \& Ekr. Lk, Le, Li. ibid.:17.

- lacustris Säw. Ok-Li. ibid.:17.

- notescens (Walk.) Al-Li. ibid.:17.

- rilensis Giłka $A b, N$, St, Lk. ibid.:17.

- schrankelae Stur \& Ekr. Lk, Le, Li. ibid.:17.

* - appendica St. \& Ekr. Lk: Sodankylä Kotaoja (M. Hirvenoja). Stur \& Ekrem 2006 Zool. J. Linn. Soc. 146:176.

* - klinki St. \& Ekr. Ta: Riihimäki (J. Tuiskunen). ibid.:193.

- pallidula (Meig.) (bidentata auct. nec (Goetgh.)). ibid.:201.
* - sofiae St. \& Ekr. Ks: Oulanka (F. Reiss). ibid.:209.

* - malla Giłka \& Paasiv. Le, Li. Giłka \& Paasivirta 2008 EF 19:42.

Neozavrelia cuneipennis (Edw.) St, Sa, Kb. Paasivirta 2009:17.

Parapsectra chionophila (Edw.) Ka-Li. ibid.:17.

Paratanytarsus abiskoensis Reiss \& Säw. Li. ibid.:18.

- bituberculatus (Edw.) St. ibid.:18.

- dimorphis Reiss $O b, L k$. ibid.:18.

* - paralaccophilus Giłka \& Paasiv. Li: Inari. Giłka \& Paasivirta 2008:44.

Rheotanytarsus curtistylus (Goetgh.) Ab-Li. Paasivirta 2009:18

- muscicola Thien. Ab-Li. ibid.:18.

- pentapoda (Kieff.) Ab-Li. ibid.:18.

Stempellinella flavidula (Edw.) Ob. ibid.:18.

- saltuum (Goetgh.) Ka. ibid.:18.

Tanytarsus anderseni Reiss \& Fittk. Li. ibid.:18.

- desertor Giłka \& Paasiv. Ab-Om. ibid.:19.

- gibbosiceps Kieff. Le. ibid.:19.

- heusdensis Goetgh. Ab-Li. ibid.:19.

- mancospinosus Ekr. \& Reiss Ka, St, Ks. ibid.:19.

- trux Giłka \& Paasiv. Li. ibid.:19.

* - paraniger Giłka \& Paasiv. Tb, Lk, Li. Giłka \& Paasivirta 2008:45.

* - salmelai Giłka \& Paasiv. Lapland. Giłka \& Paasivirta 2009 Zootaxa 2197:32.

Culicoides pseudoheliophilus Call. \& Kr. Finland. Huldén, Huldén \& Lahtinen 2008 Suom. Eläinlääk.1. 114:161.

- subfasciipennis Kieff. Finland. ibid.:161.

- sphagnumensis Will. Finland. ibid.:161.

- dewulfi (Goetgh.) Al: Kastelholm (T. Lahtinen). Hildén et al. 2010:87.

Bolitophila caspersi Plassm. Kb: Ilomantsi; Ok: Kuhmo. Polevoi et al. 2006 EF 17:163.

Pyratula perpusilla (Edw.) Ok: Kuhmo. ibid.:164.

Macrocera estonica Landr. $K b$ : Ilomantsi. ibid.:164.

- nigricoxa Winn. Kb: Ilomantsi. ibid.:164.

- pusilla Meig. Kb: Ilomantsi. ibid.:164.

Diadocidia fissa Zaitz. Kb: Ilomantsi. ibid.:163.

Manota unifurcata Lundstr. Ta: Lammi. Jakovlev \& Penttinen 2007 EF 18:214.

Mycomya fuscata Winn. Ok: Kuhmo. Polevoi et al. 2006:167.

- livida (Dziedz.) Ta: Lammi. Jakovlev et al. 2006 Sahlbergia 11:26.

- norna Väis. St, Ta, Ok. Polevoi et al. 2006:167.

- parva (Dziedz.) Ab: Karjalohja. Jakovlev et al. 2006:26.

Neoempheria bimaculata (Ros.) Ab: Karjalohja, Ta: Lammi. ibid.:26.

- winnertzi Edw. Ok: Sotkamo, Kuhmo. Polevoi et al. 2006:167 
Drepanocerus spinistylis Söli Ta: Lammi. Jakovlev et al. 2006:26.

Neuratelia sintenisi Lacks. St: Parkano; Kb: Ilomantsi. Polevoi et al. 2006:167.

Phthinia setosa Zaitz. Kb: Ilomantsi. ibid.:167.

- winnertzi Mik Ta: Lammi \& Padasjoki. Jakovlev et al. 2006:26.

Sciophila karelica Zaitz. Ta: Lammi. ibid.:26.

- minuta Zaitz. Ta: Lammi. ibid.:26.

- modesta Zaitz. Kb: Ilomantsi. Polevoi et al. 2006:167.

- nonnisilva Huts. Kb: Ilomantsi. ibid.:167.

- persubtilis Polev. Kb: Ilomantsi. ibid.:167.

- plurisetosa Edw. $N$ : Tuusula. Jakovlev \& Penttinen 2007:216.

- pseudoflexuosa Kur. Ab: Karjalohja. Jakovlev et al. 2006:26.

- setosa Garr. Ta: Lammi. ibid.:26.

Boletina bidenticulata Sas. \& Kim. Ta: Lammi, Ok: Sotkamo. Zaitzev et al. 2006 Stud. Dipterol. $12: 245$.

- gusakovae Zaitz. Ta: Lammi, Ok: Kuhmo. ibid.:247.

- nitida Grzeg. Ta: Lammi, Ok: Kuhmo. ibid.:250.

- cornuta Zaitz. Ta: Lammi, Padasjoki. Jakovlev et al. 2006:27.

- digitata Lundstr. $K b$ : Ilomantsi; $O b$ : Uleåborg. Polevoi et al. 2006:164.

- jamalensis Zaitz. Ka, St, Kb, Ok. ibid.:164.

- kurilensis Zaitz. Ta: Lammi, Li: Utsjoki. Jakovlev et al. 2006:27.

- minuta Polev. Ta: Lammi. ibid.:27.

- pallidula Edw. Ta, Kb, Ok. ibid.: Polevoi et al. 2006:164.

- takagii Sas. \& Kim. Li: Utsjoki (R. Frey). Jakovlev et al. 2006:27.

- tiroliensis Plassm. Ok: Sotkamo \& Kuhmo. Polevoi et al. 2006:164.

- triangularis Polev. Ta. Lammi. Jakovlev et al. 2006:27.

* - dispectoides Jak. \& Pent. Ta: Lammi, Tb: Keuruu. Jakovlev \& Penttinen 2007:213.

Ectrepesthoneura tori Zaitz. \& Ökl. Kb: Ilomantsi. Polevoi et al. 2006:165.

Leia cylindrica Winn. Ab: Karjalohja, Ta: Lammi \& Padasjoki. Jakovlev et al. 2006:27.

Tetragoneura sylvatica (Curt.) Kb: Ilomantsi; Ok: Kuhmo. Polevoi et al. 2006:165.

Dynatosoma silesiacum Ševč. Ta: Lammi, Padasjoki. Jakovlev et al. 2006:33.

Epicypta fumigata (Dziedz.) Ta: Lammi \& Padasjoki. ibid.:33.

- scatophora (Perr.) Ta: Lammi. ibid.:33.

* Phronia avidoides Jak. \& Pol. Ta, Tb, Kb. Jakovlev \& Polevoi 2009 EF 19:200.

* - fennica Jak. \& Pol. Ta: Lammi. ibid.:202.
Mycetophila exstincta Loew Ta: Lammi. Jakovlev et al. 2006:33.

- gibbula Edw. Ta: Padasjoki. ibid.:33.

- idonea Lašt. Ta: Lammi. ibid:33.

- morata Zaitz. Kb: Ilomantsi; Ok: Kuhmo. Polevoi et al. 2006:166.

- nigrofusca Dziedz. Ab: Karjalohja, Ta: Lammi. Jakovlev et al. 2006:34.

- ostentanea Zaitz. Kb: Ilomantsi. Polevoi et al. 2006: 166.

- pecinai Lašt. Kb: Ilomantsi; Ok: Kuhmo. ibid.:166.

- ruficollis Meig. Ab: Karjalohja, Ta: Lammi. Jakovlev et al. 2006:34.

- stolida Walk. Ab: Karjalohja, Ta: Lammi. ibid.:34.

- subsigillata Zaitz. Ab: Karjalohja, Ta: Lammi. ibid.:34.

- triangularis Lundstr. Ta: Lammi. ibid.:34.

- unguiculata Lundstr. Ta: Lammi. ibid.:34.

- xanthopyga Winn. Ab: Karjalohja, Ta: Lammi \& Padasjoki. ibid.:34.

Sceptonia demeijerei Bech. Ta: Lammi. ibid.:35.

- hamata Ševč. Ta. Lammi. ibid.:35.

- longisetosa Ševč. Ta: Lammi \& Padasjoki. ibid.:35.

- pughi Chandl. Ta: Lammi. ibid.:35.

- regni Chandl. Ta: Lammi \& Padasjoki. ibid.:35.

- thaya Ševč. Ta: Lammi. ibid.:35.

Mycetophila forcipata Lundstr. Ta: Lammi, Tb: Laukaa. Jakovlev \& Penttinen 2007:215.

- sumavica (Lašt.) Tb: Rautalampi \& Toivakka. ibid.:215.

Trichonta apicalis Strobl Kb: Ilomantsi. Polevoi et al. 2006:166.

- eximia Gagné Ta: Ruovesi; Kb: Ilomantsi. ibid.:166.

- nigritula Edw. Kb: Ilomantsi. ibid.:166.

- subterminalis Zaitz. \& Menz. Kb: Ilomantsi. ibid.:166.

- tristis (Strobl) Ab, N, Ta, Tb. Jakovlev \& Penttinen 2007:215.

Zygomyia angusta Plassm. Ta: Lammi. Jakovlev et al. 2006:35.

- notata (Stann.) Ta: Lammi \& Padasjoki. ibid.:35.

- zaitzevi Chandl. Ab: Karjalohja, Ta: Lammi \& Padasjoki. ibid.:36.

Allodia angulata Lundstr. Kb: Ilomantsi. Polevoi et al. 2006:165.

- subpistillata Ševč. Ta: Lammi. Jakovlev et al. 2006:27.

Allodiopsis gracai Ševč. \& Papp Ab, Ta, Ok, Ob. ibid.:28.

- pseudodomestica (Lacks.) Ta: Lammi. ibid.:28.

Anatella ankeli Plassm. Ok: Kuhmo. Polevoi et al. 2006:165.

- dampfi Landr. [as damfi] Ta: Lammi. Jakovlev et al. 2006:28.

- dentata Zaitz. Ta: Lammi. ibid.:28. 
- emergens Casp. Ab: Karjalohja, Ta. Lammi. ibid.:28.

- unguigera Edw. Ta: Lammi. ibid.:28.

Brachypeza obscura Winn. Kb: Ilomantsi; Ok: Kuhmo. Polevoi et al. 2006:165.

Brevicornu arcticoides Casp. Ta: Lammi. Jakovlev et al. 2006:28.

- fasciculatum (Lacks.) Ab: Karjalohja, Ta: Padasjoki. ibid.:28.

- melanderi Zaitz. Ta: Lammi. ibid.:29.

- occidentale Zaitz. Kb: Ilomantsi. Polevoi et al. 2006:165.

- parafennicum Zaitz. Ta: Lammi. Jakovlev et al. 2006:29.

Cordyla insons Lašt. \& Mat. Ab: Karjalohja, Ta: Lammi \& Padasjoki. ibid.:29.

- murina Winn. Ab: Karjalohja, Ta: Lammi \& Padasjoki. ibid.:29.

Exechia dentata Lundstr. Ab: Karjalohja \& Vihti. ibid.:29.

- pseudofestiva Lacks. Ab: Karjalohja, Ta: Lammi. ibid.:29.

- repandoides Casp. Ta: Lammi. ibid.:29.

- similis Lašt. \& Mat. Kb: Ilomantsi. Polevoi et al. 2006:165.

- subfrigida Lašt. \& Mat. Kb: Ilomantsi; Ok: Kuhmo. ibid.: 165 .

- bicincta Staeg. N: Espoo. Jakovlev \& Penttinen 2007:215.

Exechiopsis aemula Plassm. Al, Ab, N, Ka, Ta, Ok. Jakovlev et al. 2006:29.

- dumitrescae (Burgh.-Bal.) Ks: Kuusamo (R. Tuomikoski), $L i$ : Inari (R. Tuomikoski). ibid.:30.

- forcipata (Lacks.) Ab, Ta, Kb, Ok. ibid.:30.

- grassatura (Plassm.) Ab, Kb, Ks, Li. ibid.:30.

- pseudindecisa Lašt. \& Mat. St, Ta, Kb, Ok. Polevoi et al. 2006:165.

- davatchii Mat. Ta: Lammi. Jakovlev et al. 2006:31.

- membranacea (Lundstr.) (sp. dist.) Ab, Ka, Ta, Sa, Ks. ibid.:31.

- praedita (Plassm.) Ok: Sotkamo (A.V.V. Mikkola). ibid.:31.

- seducta (Plassm.) Ab: Karjalohja, Ta: Lammi \& Padasjoki. ibid.:31.

- stylata Lašt. \& Mat. Ks: Kuusamo (R. Tuomikoski). ibid.:31.

Notolopha brachycera (Zett.) N, Ta, Lk. ibid.:31.

- sibirica Zaitz. \& Max. Lk: Kittilä (R. Tuomikoski), Sodankylä (K. Keynäs). ibid.:32.

Pseudexechia canaliculata (Joh.) N: Tvärminne (K. Keynäs). ibid.:32.

- tristriata (Stack.) Ab, N. Ta. ibid.:32.

Pseudorymosia fovea (Dziedz.) Al, Ab, Ta, Sa. ibid.:32.

Rymosia acta Dziedz. N: Hangö Russarö, Ta: Lammi. ibid.:32.
- batava (Barendr.) N: Esbo (W. Hackman). ibid.:33. - pinnata Ostrov. Ta: Ruovesi. Polevoi et al. 2006:165. Synplasta dulcia (Dziedz.) Kb: Tohmajärvi (R. Tuomikoski). Jakovlev et al. 2006:33.

- ingeniosa (Kidd) Ab: Karjalohja, Ta: Lammi. ibid.:33.

Trichosia confusa Menz. \& Mohr. Ta: Urjala. Vilkamaa et al. 2007 EF 18:228.

Leptosciarella fuscipalpa (Mohr. \& Mam.) Ab: Vihti (R.Tuomikoski); Ta: Urjala. Mohrig \& Menzel 1997 Stud. dipterol. 4:65; Vilkamaa et al. 2007:228.

- juniperi (Mohr. \& Bl.-Z.) Ta: Urjala. Vilkamaa et al. 2007:228.

- rejecta (Winn.) Ta: Urjala. ibid.:228.

- subcoarctata Mohr. \& Menz. Ta: Urjala. ibid.:228.

- yerburyi (Freem.) Ta: Urjala. ibid.:228.

Schwenckfeldina pectinea Menz. \& Mohr. Al: Saltvik (R. Frey). Menzel \& Mohrig 2000 Stud. Dipterol. Suppl. 6:513.

*Corynoptera alneti Hippa et al. Ab-Li. Hippa, Vilkamaa \& Heller 2010 Zootaxa 2695:25.

* - ninae Hippa et al. Ab, Tb, Sb, Ob. ibid.:28.

- breviformis Mohr. \& Kriv. Ab, N, Oa, Li. ibid.:63.

* - plusiochaeta Hippa et al. Lk, Le, Li. ibid.:75.

- subtetrachaeta Komar. Ab, Ta, Om. ibid.:77.

- voluptuosa Mohr. \& Mam. Ks: Kuusamo (R. Tuomikoski). ibid.:85.

- melanochaeta Mohr. \& Menz. Al-Ob. ibid.:87.

- waltraudis Mohr. \& Mam. Ks: Kuusamo (J. Salmela). ibid.:91.

* - tumidula Hippa et al. Ab, Kl, Kb, Ks. ibid.:103.

- marinae Mohr. \& Kriv. Tb-Li. ibid.:144.

- polana Rudz. Ab-Li. ibid.:146.

- bipartita Mohr. \& Kriv. Ab-Ob. ibid.:150.

- postforcipata Rudz. Ta: Urjala. Vilkamaa et al. 2007:228.

Camptochaeta fallax H. \& Vilk. Ta: Urjala. ibid.:228.

- bournei (Shaw) Le: Enontekiö. Haarto 2009 Metsähall. luonnonsuoj.j. A 185:23.

Lycoriella brevipila Tuomik. (sp. dist.). Finland. Menzel \& Heller 2006 Stud. Dipterol. 13:220.

Epidapus atomarius (DeG.) Ta: Urjala. Vilkamaa et al. 2007:228.

Phytosciara macrotricha (Leng.) Ta: Urjala. ibid.:228.

Bradysia excelsa Menz. \& Mohr. Ta: Urjala. ibid.:228.

* - arcula Vilk., Salm. \& H. Ta, Tb, Ob. ibid.:229.

- moestula Tuomik. (sp.dist.). Heller et al. 2009 Sahlbergia 15:28.

Allarete nigra Mam. Lk: Kittilä. Jaschhof \& Jaschhof 2009 Stud.dipterol., Suppl. 18:62.

Anaretella iola Pritch. St, Ob, Ks. ibid.:66.

Lestremia cinerea Macq. Al, Ab, N, St, Ta, Sa, Kl, Tb, $K b, O k, O b, K s, L k$, Li. ibid.: 72 .

*Catocha incisa Jaschh. N, St, Ta, Sa, Tb, Kb. ibid.:79. 
- latipes Halid. St, Tb, Kb. ibid:81.

Acoenonia europaea Mam. Kb: Lieksa, Ok: Kuhmo ibid.:89.

Campylomyza alpina Sbke. Al, St, Kl, Tb, Ok, Ks, Lk. ibid:92.

- dilatata Felt Ta: Tammela, Kb: Outokumpu. ibid.:93.

- fusca Winn. Ks: Kuusamo. ibid.:93.

* - stegetfore Jaschh. St, Ta, Sa, Tb, Kb, Ok, Ks. ibid.:94.

- armata Mam. Ta: Tammela, Tb: Saarijärvi. ibid.:96.

* - insolita Jaschh. Tb: Saarijärvi ibid.:99.

- aemula Mam. St: Ikaalinen, Ta: Lammi. ibid.:101.

* - falcifera Jaschh. Ab: Vihti, St: Huittinen. ibid.:105.

- furva Edw. Kb: Lieksa, Ks: Kuusamo. ibid.:107.

- abbreviata Jaschh. Sb: Joroinen. ibid.:110

- cavitata Mam. Kb: Lieksa. ibid.:113.

* - inornata Jaschh. Kl: Parikkala. ibid.:114

- ormerodi (Kieff.) $A b, N, T a, T b, K b$. ibid.:115.

- serrata Jaschh. Ab, N, St, Ta, Sa, Kb, Ks. ibid.:117.

* - arcuata Jaschh. Kb: Lieksa. ibid.:118.

* - cingulata Jaschh. St, Ta, Tb, Kb, Ks, Lk. ibid.:119.

- spatulata Mam. Sa: Lappeenranta. ibid.:122.

Excrescentia mutuata Mam. \& Ber. Ab: Turku, Tb: Saarijärvi. ibid.:123.

*Neurolyga acuminata Jaschh. St, Sa, Tb. ibid.:126.

- bilobata (Mam. \& Rozh.) Tb: Saarijärvi, Kb: Lieksa. ibid.:129.

- excavata (Yuk.) Lk: Kittilä. ibid.:131.

* - interrupta Jaschh. Lk: Kittilä. ibid.:132.

* - lonsdalensis Jaschh. Sa: Taipalsaari. ibid.:133.

- sylvestris (Felt.) Sa: Lappeenranta. ibid.:138.

- verna (Mam.) St: Huittinen. ibid.:139.

*Peromyia abdita Jaschh. Ob: Pudasjärvi. ibid.:143.

- bicolor (Edw.) St, Ta, Tb, Kb, Ok, Ob, Ks, Lk. ibid.:144.

- bidentata Ber. Kl: Parikkala. ibid.:145.

- scutellata Mam. Ab, St, Tb, Kb, Lk. ibid.:146.

* - subbicolor Jaschh. Lk: Kittilä. ibid.:147.

- syltefjordensis Jaschh. St, Kb, Ok. ibid.:148.

- subborealis Jaschh. Al, Kl. ibid.:149.

- angellifera Jaschh. Kl: Parikkala. ibid.:150.

- caricis (Kieff.) N, St, Ta, Sa, Kl, Tb, Kb, Ok, Ks, Lk. ibid.: 150 .

- diadema Mam. Ab, St, Ta, Sa, Kl, Kb. ibid.:151.

- fagiphila Jaschh. Kl: Parikkala. ibid.:152.

- pumila Jaschh. Kl: Parikkala. ibid.:152.

* - semotoides Jaschh. Kl: Parikkala. ibid.:152.

- cornuta (Edw.) Ab: Kimito, Ks: Kuusamo. ibid.:154.

- ramosa (Edw.) Lk: Kittilä. ibid.:155.

* - ramosoides Jaschh. St: Ikaalinen. ibid.:155.

- curta Jaschh. Ab, N, St, Ta, Sa, Tb, Kb. ibid.:157.

- anatina Mam. \& Ber. Ab: Turku. ibid.:157.

- intermedia (Kieff.) Tb, Kb, Ok, Ob. ibid.: 158.

- anisotoma Mam. Ta: Tammela. ibid.:159.

- boreophila Jaschh. Kb: Kontiolahti. ibid.:161.
- concitata Mam. \& Ber. St, Ta, Kb. ibid.:163.

- modesta (Felt) Ab: Västanfjärd, Ok: Kuhmo. ibid.:165.

- ovalis (Edw.) Al: Eckerö. ibid.:166.

- perpusilla (Winn.) Ab, St, Ta, Tb, Kb, Ok, Ob, Ks, Lk. ibid.:167.

- tundrae Jaschh. Ks: Kuusamo. ibid.:167.

- abnormis Mam. \& Ber. Tb, Kb, Lk. ibid.:168.

- monilis Mam. St, Kl, Tb, Ok, Lk. ibid.:170.

* - scirrhosa Jaschh. Tb, Ok, Ks. ibid.:170.

- edwardsi Ber. St: Ikaalinen, Ta: Tammela. ibid.:172.

- fungicola (Kieff.) $\mathrm{St}, \mathrm{Ta}, \mathrm{Sa}, \mathrm{Kl}, \mathrm{Tb}, \mathrm{Kb}, \mathrm{Ok}, \mathrm{Ob}, \mathrm{Ks}$, $L k$. ibid.: 172 .

- muscorum (Kieff.) $\mathrm{Al}, \mathrm{Kl}, \mathrm{Tb}, \mathrm{Kb}, \mathrm{Ok}, \mathrm{Ob}, \mathrm{Ks}, \mathrm{Lk}$. ibid.: 173 .

- palustris (Kieff.) Ta, Kb, Ok. ibid.:174.

- photophila (Felt) N, St, Ta, Kl, Kb, Ok, Ks. ibid.:174.

- truncata Yuk. St: Ikaalinen, Kb: Lieksa. ibid.:175.

- upupoides Jaschh. St, Ta, Tb. ibid.:176.

- apposita Jaschh. St, Ta, Kl, Tb, Kb, Ok. ibid.:176.

- mitrata Jaschh. St: Ikaalinen. ibid.:178.

- sanguinea (Kieff.) Ab, Ta, Ok. ibid.:178.

- albicornis (Meig.) Ks: Kuusamo. ibid.:180.

Bryomyia bergrothi Kieff. $\mathrm{Al}, \mathrm{N}, \mathrm{St}, \mathrm{Ta}, \mathrm{Sa}, \mathrm{Kl}, \mathrm{Tb}, \mathrm{Kb}$, $O k, O b, K s, L k$. ibid.: 184.

- gibbosa (Felt) Ab, St, Ta, Sa, Kl, Tb, Kb, Ok, Ks, Li. ibid.: 185 .

- producta (Felt) N, Tb, Ks, Lk. ibid.:186.

Heterogenella finitima Mam. Ta: Lammi, Kl: Parikkala. ibid.:189.

- hybrida Mam. St, Ta, Kl, Tb, Kb, Ok, Ob, Ks, Lk. ibid.:189.

- linearis Yuk. St, Tb, Kb, Ok, Ob. ibid.:190.

Skuhraviana triangulifera Mam. Ab, St, Kl, Kb, Ok. ibid.:193.

Micromya lucorum Rond. Ab: Vihti. ibid.:195.

Monardia abnormis Mam. Ab, Kl, Tb, Kb. ibid.:198.

- armata Jaschh. St: Yläne, Ok: Puolanka. ibid.:198.

- lignivora (Felt) $A b$ : Vihti. ibid.:199.

- obsoleta Edw. Al, Ab, St, Ta, Ok, Ks, Lk. ibid.:201.

- pediculata (Mam.) N, Sb, Ok. ibid.:202.

- stirpium Kieff. St, Kb, Ok. ibid.:203.

- yasumatsui Yuk. Kb: Lieksa. ibid.:204.

- magnifica Mam. St, Tb, Kb, Ks, Lk. ibid.:205.

* - relicta Jaschh. St: Ikalinen, Tb: Saarijärvi. ibid.:208.

- adentis Jaschh. Tb: Saarijärvi. ibid.:209.

- atra (Meig.) Sa, Tb, Ok, Ob, Ks, Lk, Li. ibid.:210.

- monotheca Edw. Ta, Sa, Tb, Ks. ibid.:211.

- radiella Mam. Ok, Ob, Ks. ibid.:212.

- toxicodendri (Felt) Ab: Vihti. ibid.:213.

- unguifera Ber. \& Mam. Sa: Savonlinna. ibid.:214.

Polyardis adela Pritch. Sa: Lappeenranta. ibid.:217.

- bispinosa (Mam.) Ab, Sa, Kl, Tb, Kb. ibid.:217. 
- silvalis (Rond.) Sa: Lappeenranta, Kl: Parikkala. ibid.:218.

Aprionus acutus Edw. Kb: Lieksa, Ok: Kuhmo. ibid.:222.

- subacutus Jaschh. St, Ta, Kb, Ks. ibid.:222.

* - brevitegminis Jaschh. Lk: Kittilä. ibid.:223.

* - latitegminis Jaschh. Lk: Kittilä. ibid.:225.

- longitegminis Yuk. Ta: Lammi. ibid.:227.

- marginatus Mam. Tb: Saarijärvi, Kb: Lieksa. ibid.:227.

- accipitris Jaschh. Ab: Västanfjärd. ibid.:229.

- aviarius Mam. \& Ber. $K l, T b, K b, O k$. ibid.:230.

- betulae Jaschh. St, Ta, Kl, Tb, Kb, Ok, Ob, Lk. ibid.:232.

- bidentatus (Kieff.) Al: Eckerö. ibid.:232.

- bifidus Mam. Ab, St, Ta, Ks, Lk. ibid.:233.

- bispinosus Edw. Al, Kl, Kb, Ok, Ob, Ks, Lk. ibid.:233.

- carinatus Jaschh. Al, Ab, N, St, Ta, Sa, Kl, Tb, Kb, Ok, $O b, K s, L k$. ibid.:234.

- dissectus Mam. \& Ber. Kb, Ok, Ks. ibid.:238.

- duplicatus Mam. St, Ta, Kl, Tb, Kb, Ok, Lk. ibid.:238.

-flavidus (Winn.) Ab, St, Tb, Kb, Ok, Ks, Lk. ibid.:240.

- stylifer Mam. Ab, N, Ta, Tb, Kb, Ok, Ks. ibid.:242.

- brachypterus Edw. St, Sa, Kl, Tb, Kb, Ok, Ob, Ks, Lk, Li. ibid.:243.

- styloideus Mam. \& Ber. St, Sa, Tb, Kb, Lk. ibid.:244.

- halteratus (Zett.) Ta, Sa, Kl, Ok, Lk. ibid.:245.

- piceae Jaschh. Kb, Ok, Ks. ibid.:245.

* - foliosus Jaschh. Ob: Pudasjärvi. ibid.:246.

- insignis Mam. St, Ta, Tb, Kb, Ok, Lk. ibid.:247.

- longicollis Mam. Ta, Kl, Tb, Kb. ibid.:248.

- miki Kieff. St, Kb, Ok, Ks, Lk. ibid.:249.

* - taigaensis Jaschh. Kb: Lieksa, Ob: Puolanka. ibid.:249.

- dalarnensis Mam. St, Tb, Kb, Ok. ibid.:252.

* - oligodactylus Jaschh. St, Ta, Sa, Kb. ibid.:252.

- separatus Mam. \& Jaschh. Ab, St, Ta, Kl, Tb, Ob, Ks. ibid.:254.

* - fennicus Jaschh. Tb: Saarijärvi, Kb: Lieksa. ibid.:254.

- arcticus Mam. St, Ta, Sa, Tb, Ks, Lk. ibid.:257.

- cornutus Ber. St, Ta, Tb, Kb, Ok, Ks. ibid.:257.

- ensiferus Jaschh. Lk: Kittilä. ibid.:257.

* - gladiator Jaschh. Tb, Ks, Lk. ibid.:258.

- inquisitor Mam. N, St, Ta, Tb, Ok, Ob, Ks, Lk. ibid.:259.

- lapponicus Jaschh. \& Mam. Ab, N, St, Ta, Sa, Tb, Kb, $O b, K s, L k$. ibid.:260.

* - sievertorum Jaschh. Ta: Tammela. ibid.:260.

* - victoriae Jaschh. Kb: Lieksa. ibid.:261.

- cardiophorus Mam. Al, St, Ta, Tb, Ks, Lk. ibid.:263.

- complicatus Mam. \& Ber. N, Ta, Sa. ibid.:264.

- dispar Mam. Tb: Saarijärvi, Ok: Kuhmo. ibid.:265.

* - hintelmannorum Jaschh. St: Ikaalinen, Kb: Lieksa. ibid.:265.
- laevis Mohr. Al, Ta, Sa, Tb. ibid.:268.

* - reduncus Jaschh. Kb: Lieksa. ibid.:268.

- spiniferus Mam. \& Ber. St, Kb, Ok, Ks, Lk. ibid.:271.

* - stiktos Jaschh. Lk: Kittilä. ibid.:271.

- tiliamcorticis Mam. Ab, N, St, Tb, Kb, Ok, Lk. ibid.:273.

* - adventitius Jaschh. St, Ta, Tb, Kb, Ok, Lk. ibid.:275.

* - aquilonius Jaschh. Tb, Kb, Ok. ibid.:277.

* - heothinos Jaschh. Kb: Lieksa, Ok: Kuhmo. ibid.:279.

- similis Mam. St, Ta, Tb, Kb, Ks, Lk. ibid.:281.

- spiniger (Kieff.) N, St, Ta, Sa, Kl, Tb, Kb, Ok, Ob, Ks, Lk. ibid.:282.

- corniculatus Mam. Ab, St, Tb, Kb, Ok, Ob, Ks, Lk. ibid.:283.

- dentifer Mam. St, Sa, Tb, Sb, Kb, Lk. ibid.:283.

- laricis Mam. \& Jaschh. Kb: Lieksa. ibid.:284.

* - praecipuus Jaschh. St: Ikaalinen, Ok: Kuhmo. ibid.:285.

- pyxidiifer Mam. $K l, T b, K b, O k, L k$. ibid.:287.

- svecicus Jaschh. Ta, Kb, Ok, Ob, Ks, Lk. ibid.:288.

Holoneurus paneliusi Yuk. Le: Kilpisjärvi (J. Jakovlev \& J. Penttinen). Penttinen \& Spungis 2007 Sahlbergia 12:39.

Schistoneurus irregularis Mam. Kb: Kontiolahti (M. Jaschhof), Ks: Kuusamo (M. Jaschhof). ibid.:39.

Coccopsilis marginata (Meij.) Ab: Turku (M. Jaschhof). ibid.:39.

Parepidosis venustior Gagné Ok: Sotkamo (M. Jaschhof). ibid.:39.

Porricondyla globosa Spung. Ok: Sotkamo, Lk: Kolari \& Kittilä. ibid.:40.

Spungisomyia media (Spung.) Lk: Kolari \& Kittilä. ibid.:40.

Asynapta strobi (Kieff.) Ks: Kuusamo, Lk: Kolari. ibid.:38.

Camptomyia pinicola Mam. Tb: Laukaa (J. Penttinen). ibid.:38.

Diallactes obscuripes Spung. Tb: Keuruu (J. Penttinen), ibid.:38.

Dicerura furculata Mam. Ab: Turku (M. Jaschhof). ibid.:39.

- mixta Spung. Ab: Turku (M. Jaschhof). ibid.:39.

Solntsevia nigripes Mam. Ks: Posio (M. Jaschhof). ibid.:40.

Winnertzia globifera Mam. Ok: Sotkamo (M. Jaschhof). ibid.: 40 .

- graduata Spung. Tb: Saarijärvi, Ok: Kuhmo (M. Jaschhof). ibid.: 40 .

- nigripennis (Kieff.) Ok: Kuhmo, Lk: Kolari. ibid.:41.

Sylvenomyia spinigera (Spung.) Ta: Lammi, Sb: Savonranta, $K b$ : Lieksa. Penttinen \& Jaschhof 2009 Zootaxa 2032:52.

* - fennica Pent. \& Jsch. St, Ta, Tb, Kb, Ok, Ob, Lk. ibid:52. 
Sciapus albifrons (Meig.) Kl: Parikkala (W. Hellén). Kahanpää \& Grichanov 2006 EF 17:76.

- basilicus Meuff. \& Gr. N: Tvärminne (A. Wegelius). ibid.:76.

- zonatulus (Zett.) N: Tvärminne, Lappvik \& Porvoo. ibid.:76.

Rhaphium albifrons Zett. Le: Kilpisjärvi (J: Kahanpää). ibid.:76.

- fissum Loew Ab, Sb, Ok, Ob. Kahanpää 2006 Dipterol. Res. 17:122.

Chrysotimus molliculus (Fall.) $N$ : Helsinki; Sa: Ristiina. Kahanpää \& Grichanov 2006:74.

Achalcus vaillanti Brunh. N, Om. ibid.:74.

Peodes petsamoensis Frey Li: Utsjoki. Kahanpää 2006:122.

Syntormon freymuthae Loew $A b, N, S t, T a, S a, T b$. ibid.: 123.

Teuchophorus monacanthus Loew $N$ : Mäntsälä. Kahanpää \& Salmela 2007 Sahlbergia 12:46.

Telmaturgus tumidulus (Radd.) N: Kyrkslätt; Ta: Hattula. Kahanpää \& Grichanov 2006:77.

Lamprochromus strobli Par. $N$, Ka, Sb. ibid.:76.

Argyra ilonae Goss. Al, Ab, N, Sa. ibid.:74.

- setimana Loew $A b, K a$, Sb. ibid.:74.

Diaphorus hoffmannseggi Meig. Ab: Lojo (W. Hellén). ibid.:75.

Chrysotus angulicornis Kow. $A b$ : Vichtis (R. Frey); $N$ : Hangö (R. Frey). ibid.:74.

Tachytrechus hamatus Loew Ta: Hattula (J. Kahanpää). ibid.:76.

*Nemotelus infortunatus Kahanp. Sb, Kb. Kahanpää 2010 Zootaxa 2401:30.

Hercostomus nigriplantis (Stann.) N: Hanko. Kahanpää 2006:122.

Dolichopus apicalis Zett. N, Sa. Kahanpää \& Grichanov 2006:75.

- calinotus Loew N: Helsinki (J. Kahanpää). ibid.:75.

- cinctipes Wahlb. Lk: Muonio (R. Frey). ibid.:75.

- clavipes Hal. St: Eura (R. Storå). ibid.:75.

- hilaris Loew N: Helsinki. Kahanpää 2006:122.

- latilimbatus Macq. N: Helsinki (J. Kahanpää). Kahanpää \& Grichanov 2006. 75.

- signifer Hal. Ab: Korpo Jurmo (M. Meinander). ibid.:75.

- subpennatus d'Ass. Ab, N, Ta, Sa. ibid.:75.

Sybistroma obscurellum (Fall.) N: Inkoo \& Espoo. Kahanpää 2006:123.

Systenus scholtzii (Loew) Al: Lemland (D. Rotheray). Kahanpää \& Grichanov 2006:76.

[Medetera truncorum Meig.] Ab: Turku (V. Rinne). Kahanpää 2006:124.

Thrypticus intercedens Negr. $\mathrm{Al}, \mathrm{Ab}, \mathrm{N}, \mathrm{Ka}, \mathrm{Sa}, \mathrm{Om}$. Kahanpää \& Grichanov 2006:77.

- nigricauda Wood Ab: Karislojo (R. Frey). ibid.:77.

- tarsalis Par. Ab, N, Oa. Kahanpää 2006:123.
* Megaselia excorticata Disn. Ok: Sotkamo \& Puolanka. Disney 2010 EF 20:258.

- longifurca (Lundb.) Ok: Kuhmo \& Sotkamo. ibid.:261.

- robertsoni Disn. Ok: Sotkamo. ibid.:262.

- setulipalpis Schm. Ok: Kuhmo. ibid.:262.

* - tignorum Disn. Ok: Kuhmo \& Puolanka. ibid.:263.

Agathomyia vernalis Shat. Ta: Hartola. Ståhls \& Kahanpää 2006 Sahlbergia 11:2

.- unicolor Oldenb. Ta: Hartola. ibid.:3.

- lundbecki Chandl. Ab: Karjalohja. ibid.:3.

- wankowiczii (Schnabl) N: Helsinki, Ta. Lammi. ibid.:3.

- zetterstedti (Wahlb.) Ab: Karjalohja. ibid.:3.

Platypeza consobrina Zett. Ta: Hämeenlinna. ibid.:4. - hirticeps Verr. Ta: Lammi \& Hämeenlinna. ibid.:4.

Epistrophe olgae Mut. Ab, N, Ta, Sa, Kl, Tb, Sb, Kb, Om, Ks. Haarto \& Kerppola 2007:620. Haarto \& Kerppola 2008 Sahlbergia 13(2):36.

Eupeodes biciki Niels. Le: Kilpisjärvi (R. Frey). Haarto \& Kerppola 2007:172. Haarto \& Kerppola 2008:36.

- duseki Maz., Lás. \& Bič. Le: Kilpisjärvi, Li: Utsjoki. Haarto \& Kerppola 2007:175. Haarto \& Kerppola 2008:36.

- goeldlini Maz., Lás. \& Bič. Ab, N. Ta, Om, Ob, Li. Haarto \& Kerppola 2007:620. Haarto \& Kerppola 2008:36.

- tirolensis (Duš. \& Lás.) Le: Kilpisjärvi (A. Haarto). Haarto \& Kerppola 2007:180. Haarto \& Kerppola 2008:36.

Sphaerophoria kaa Viol. Ks: Salla, Lk: Muonio, Le: Enontekiö. Haarto \& Kerppola 2007:229. Haarto \& Kerppola 2008:37.

- pallidula Mutin Kb: Ilomantsi (P. Bagge \& I. Kakko). Haarto \& Kerppola 2009 w-album 7:7.

Xanthogramma stackelbergi Viol. Al, Ab, N, Ta, Sb. Haarto \& Kerppola 2008:37.

Sphiximorpha subsessilis (Ill.) Ab, N, Ta. Haarto \& Kerppola 2007:251. Haarto \& Kerppola 2008:37.

Platycheirus magadanensis Mut. Lk: Muonio (R. Frey), Le: Kilpisjärvi (A. Nordman). Haarto \& Kerppola 2007:100. Haarto \& Kerppola 2008:35. - aurolateralis Stubbs Ab, Ta, Sa, Li. Haarto \& Kerppola 2007:89. Haarto \& Kerppola 2008:34.

- urakawensis (Mats.) Le: Enontekiö. Haarto \& Kerppola 2008:35. Haarto 2009 Metsähall. Luonnons.julk. A 185:23.

- brunnifrons Niels. $A b$ : Uskela (E.J.Bonsdorff), $N$ : Helsinki(O. Ranin). Haarto \& Kerppola 2008:34.

- goeldlini Niels. $N$ : Helsingfors (H. Ingelius), Ta: Messuby (R. Frey). Haarto \& Kerppola 2007:93. Haarto \& Kerppola 2008:35.

Paragus pecchiolii Rond. (majoranae auct.). Finland. Haarto \& Kerppola 2008:36. 
Heringia larusi Vuj. Ab, N, Ta, Sa, Kl, Sb, Om, Ok, Ob, Ks, Le. Haarto \& Kerppola 2007:630. Haarto \& Kerppola 2008:38.

- latitarsis (Egg.) N: Helsinki. Haarto \& Kerppola 2009:10.

Pipiza accola Viol. N, Ta. Haarto \& Kerppola 2007:630. Haarto \& Kerppola 2008:38.

Cryptopipiza notabila Violov. $N$, Ka, St, Ks. Ståhls \& Vujić 2009 Sahlbergia 15(2):1.

Cheilosia flavissima Beck. (pallipes auct.) $N, O a, T b$, $S b, O b, L k$. Haarto \& Kerppola 2007:624. Haarto \& Kerppola 2008:38.

* - naruska Haarto \& Kerp. Ob, Ks, Lk, Li. Haarto, Kerppola \& Ståhls 2007 Volucella 8:63. Haarto \& Kerppola 2008:37.

- reniformis Hell. N: Helsinki. Haarto \& Kerppola 2007:282. Haarto \& Kerppola 2008:38.

- vulpina (Meig.) N, Ta, Sa, Kb. Haarto \& Kerppola 2007:275. Haarto \& Kerppola 2008:38.

Brachyopa vittata (Zett.) Sa: Joutseno (S. Kerppola). Haarto \& Kerppola 2007:320. Haarto \& Kerppola 2008:38.

- zhelochovtsevi Mutin $K b$ : Ilomantsi (I. Kakko), Le: Enontekiö (R. Frey). Haarto \& Kerppola 2009:4.

Melanogaster parumplicata (Loew) Al: Jomala (H. Bartsch), $A b$ : Karjaa (A. Haarto). ibid.:9.

Xylota xanthocnema Coll. Sa: Joutseno (van Steenis \& van Zuijen). Haarto \& Kerppola 2008:39.

Chalarus argenteus Coe $A b$ : Turku (K. Winqvist). Kehlmaier \& Ståhls 2008 Sahlbergia 13(2):46.

* - immanis Kehlm. Ab: Karjalohja (Kehlmaier \& Ståhls). Kehlmaier 2008b Zootaxa 1936:7.

Jassidophaga beatricis (Coe) Ab, Ka, Ta, Sa, Kl. Kehlmaier \& Ståhls 2008:47.

Pipunculus calceatus Ros. N: Sibbo (A. Albrecht), Ok: Hyrynsalmi (J. Kahanpää). Kehlmaier 2008a Zootaxa 1672:5. Kehlmaier \& Ståhls 2008:54.

- dimi Kuzn. N: Sibbo \& Borgå (A.Albrecht). Kehlmaier 2008a:13. Kehlmaier \& Ståhls 2008:54.

- elegans Egg. $A l, A b, N$. Kehlmaier 2008a:14. Kehlmaier \& Ståhls 2008:54.

- fonsecai Coe $A b$ : Turku (K. Winqvist), $N$ : Tvärminne \& Sibbo (A. Albrecht). Kehlmaier 2008:14. Kehlmaier \& Ståhls 2008:54.

- lenis Kuzn. Al, Ab, N, Kb. Kehlmaier 2008a:16. Kehlmaier \& Ståhls 2008:54.

- lichtwardti Kozán. Ta: Sysmä (J. Kahanpää). Kehlmaier 2008a:17. Kehlmaier \& Ståhls 2008:55.

- oldenbergi Coll. N, Kl. Kehlmaier 2008:19. Kehlmaier \& Ståhls 2008:55.

- omissinervis Beck. Ab, N, Ta, Tb, Ok, Li. Kehlmaier 2008a:20. Kehlmaier \& Ståhls 2008:55.
- tenuirostris Kozán. Al, Ab, N, Sa, Sb. Kehlmaier 2008a:24. Kehlmaier \& Ståhls 2008:55.

Clistoabdominalis doczkali Kehlm. Sa: Kangaslampi (N. Laurenne). Kehlmaier \& Ståhls 2008:57.

Eudorylas angustimembranus Koz. \& Kw. Ab: Mietoinen (A. Haarto), $N$ : Sibbo (A. Albrecht). ibid.:58.

- arcanus Coe $A b$ : Mietoinen (A. Haarto). ibid.:58.

- barkalovi Kuzn. N: Sibbo, Ok: Kuhmo, Le: Kilpisjärvi. ibid.:58.

- carpathicus Koz. N: Sibbo (A. Pekkarinen \& A. Albrecht). ibid.:58.

- fascipes (Zett.) Ab: Kaarina (V. Mukkala), N: Sibbo (A. Albrecht). ibid.:58.

- inferus Coll. Ab: Mietoinen (A. Haarto), $N$ : Tvärminne (A. Albrecht). ibid.:59.

- jenkinsoni Coe $A l, A b, N, T a, O k$. ibid.:59.

- johnenae Demp. $N$ : Tvärminne (A. Albrecht), Ks: Salla (A. Haarto). ibid.:59.

- kowarzi (Beck.) Ab: Ruissalo (V. Mukkala), N: Sibbo (A. Albrecht). ibid.:59.

- montium (Beck.) Ab, N, Ta, Ob, Ks. ibid.:59.

- obscurus Coe Ab, N, Ka, Ta, Sa, Kb, Ob. ibid.:59.

- restrictus Coe Al-Ok. ibid.:60.

- slovacus Koz. N: Tvärminne(A. Albrecht). ibid.:60.

- stackelbergi Kuzn. Ab: Karjalohja, N: Sibbo. ibid.:60.

- subfuscipes Coll. Al-Ob. ibid.:60.

- subterminalis Coll. $N$ : Tvärminne (A. Albrecht \& K. Keynäs). ibid.:60.

- vonderdunki Demp. Ab: Karjalohja, N: Sibbo. ibid.:61.

- zermattensis (Beck.) Al, N, Ta, Om. ibid.:61.

- zonellus Coll. $A b, N$, Ka. ibid.:61.

Dorylomorpha aczeli Hardy Ab: Vichtis, $N$ : Sibbo. ibid.:61.

- extricata (Coll.) St: Karvia. ibid.:61.

*Tomosvaryella kalevala Kehlm. Al, Ab, N, Ta, Sb, Kb, Lk. Kehlmaier 2008a:33. Kehlmaier \& Ståhls 2008:65.

- rossica Kuzn. $N$ : Espoo, $L k$ : Pallastunturi. Kehlmaier 2008a:38. Kehlmaier \& Ståhls 2008:65.

- coquilletti (Kert.) $N$ : Vanda (A. Albrecht), $K b$ : Kontiolahti (H. Lindberg). Kehlmaier \& Ståhls 2008:65

- kuthyi Acz. Ab, N, Sa, Kb. ibid.:65.

- minuscula (Coll.) Ab: Turku (K. Winqvist). ibid.:65.

- palliditarsis (Coll.) N: Hangö \& Helsinki, Sb: Iisalmi. ibid.:65.

Thecophora jakutica Zim. Ta, Tb, Kb, Ob. Kahanpää 2007 EF 18:171.

Pseudotephritis trypetoptera (Henn.) Ab, St, Ta. Winqvist \& Kahanpää 2007:25

Melieria omissa (Meig.) (obscuripes auct.) Ab, N, Ka, St, Oa, Om, Ob. ibid.:25. 
Homalocephala biumbrata (Wahlb.) (albitarsis auct.) Finland. ibid.:30.

Campiglossa solidaginis (White) Ta: Tampere (E. \& L. Laasonen), $S a$ : Joutseno (A. Valtonen). Söderman et al. 2007 Sahlbergia 12:10.

Oxyna nebulosa Wied. Al: Jomala, Kb: Ilomantsi. ibid.:11.

Tephritis rydeni (Her.) (dioscurea auct.). Al- Ob. ibid.:7,17.

Terellia colon (Meig.) Ab: Lohja (Lindén \& Sinkkonen). ibid.:11.

- plagiata (Dahlb.) Al: Finström (A. Nordman). ibid.:12.

Euphranta toxoneura (Loew) Sa: Mikkeli, Ok: Paltamo \& Sotkamo. ibid.:3.

Chetostoma stackelbergi (Rond.) Ab, N, Ta. ibid.:4.

Cornutrypeta [Stemonocera] superciliata (Frey) $N$ : Sibbo (A. Albrecht), Ta: Somero (A. Haarto). ibid.:5.

Dryomyza decrepita Zett. (sp.dist.) Winqvist \& Kahanpää 2007:31.

Pteromicra pectorosa (Hend.) Ab: Mietoinen (A. Haarto). :25.

Sciomyza sebezhica Przh. Tb: Jyväskylä, Ob: Liminka. ibid.:25.

Palloptera modesta (Meig.) (parallela Loew) (sp.dist.). ibid.:28.

Dasiops occultus Coll. Ab: Nagu (A. Nordman). ibid.:22

Chaetolonchaea pallipennis (Zett.) (dasyops auct.). $A l, A b, N, K a$. ibid.:22.

Lonchaea albigena (Coll.) Ta: Birkkala (R. Frey), Sa: S:t Michel (Nordqvist). ibid.:22.

- limatula Coll. (flavidipennis auct.) Finland. ibid.:28.

- scutellaris Rond. Kl: Parikkala (M. Koponen). ibid.:24.

- tarsata Fall. Al: Föglö. ibid.:24.

- ultima Coll. Al: Lemland. ibid.:24.

Agromyza reptans (Fall.) Ka: Vehkalahti (L. Tiensuu). Winqvist 2007 Diamina 16:28.

Ophiomyia ranunculicaulis Her. Ka: Virolahti (L. Tiensuu), ibid.:28.

Melanagromyza angeliciphaga Spenc. Ka: Vehkalahti \& Virolahti (L. Tiensuu). ibid.:28.

Phytomyza asteris Hend. Ka: Vehkalahti (L. Tiensuu). ibid.:28.

- heracleana Her. Ka: Hamina (L. Tiensuu). ibid.:28.

- isais Her. Ka: Hamina (L. Tiensuu). ibid.:29.

- mylini Her. Ka: Vehkalahti (L. Tiensuu). ibid.:29.

- pimpinellae Hend. $N$ : Helsinki (L. Tiensuu). ibid.:29.

- rydeni Her. Ka: Vehkalahti \& Virolahti (L. Tiensuu). ibid.:29.

- selini Her. Ka: Vehkalahti (L. Tiensuu). ibid.:29.
- soenderupi Her. Ka: Vehkalahti (L. Tiensuu). ibid.:29.

- thysselini Hend. Ka: Vehkalahti (L. Tiensuu). ibid.:29.

- virosae Pakaln. Ta: Hattula (Tiensuu). ibid.:29.

Heteromyza rotundicornis (Zett.) $N$ : Mäntsälä. Kahanpää \& Salmela 2007 Sahlbergia 12:46.

Philotelma defectum (Hal.) Al, Ab, Om, Ob. Mathis, Zatwarnicki \& Kubátova-Hiršová 2009 Insect Syst. Evol. 40:133.

*Delia coarctoides Mich. $N$ : Tvärminne (R. Frey), $K a$ : Vehkalahti (L. Tiensuu), Ta: Loppi (I. Kakko); Kb: Joensuu (O. Martin). Michelsen 2007a Zootaxa 1412:63.

* - rimiventris Mich. Ab: Naantali (L. Tiensuu), Ka: Vehkalahti (L. Tiensuu). Michelsen 2007b Zootaxa 1469:53.

Paradelia lundbeckii (Ringd.) Le: Malla (R. Frey). Michelsen 2007c Zootaxa 1592:8.

- brunneonigra (Schnabl) Ka, Ta, Tb, Kb. ibid.:17.

*Pegomya curviphallis Mich. Ab: Turku (K. Winqvist), $N$ : Helsinki (L. Tiensuu). Michelsen 2006 Zootaxa 1257:51.

* - glabroides Mich. Ks: Salla (Krogerus). Michelsen 2008 Zooytaxa 1781:45.

Fannia atra (Stein) Le: Enontekiö. Haarto 2009 Metsähall. Luonnons.julk. A 185:25.

Phaonia amabilis (Meig.) Le: Enontekiö. ibid.:25.

- atrocyanea (Ringd.) Le: Enontekiö. ibid.:25.

Chetogena tschorsnigi Ziegl. Ta: Tammela (K. Mattila). Pohjoismäki 2006 Diamina 15:1.

*Phebellia margaretae Bergstr. Ob: Hailuoto (R. Frey), $T b$ : Saarijärvi (F. Woldstedt), $S b$ : Tuovilanlaks (J.A. Palmén) Idensalmi (C. Lundström). Bergström 2005 Stuttgart Beitr. Naturk. (A) 679:2.

- triseta (Pand.) Ok: Sotkamo. Pohjoismäki 2006:2.

*Loewia erecta Bergstr. Al, Ab, Ta, Ob. Bergström 2007 Stuttgart. Beitr. Naturk. (A) 708:3.

Siphona nigricans (Villen.) Li: Nuorgam. Pohjoismäki 2006.:2.

Phasia barbifrons (Girschn.) Ta: Valkeakoski. ibid.:2.

Present number: $5,551-48+709=6,212 \mathrm{spp}$.

\section{Siphonaptera}

Additions

Catallagia dacenkoi Ioff $L i$ : Inari. Brinck-Lindroth \& Smit, 2007 Fauna Ent. Scand. 41:55.

Ceratophyllus borealis Rothsch. (sp. dist.) $A l, A b, N$, $T a, S b, K b, O b$. ibid.:85, 159.

Present number: $52+2=54$ spp. 


\section{Hymenoptera}

Humala, A. 2003. Naezdniki-ihnevmonidy fauny Rossii i sopredel'nyh stran: Podsemejstva Microleptinae i Oxytorinae (Hymenoptera: Ichneumonidae). - Moskva, Nauka, 175 pp.

Taeger, A., Blank, S.M. \& Liston, A.D. 2006. European Sawflies (Hymenoptera: Symphyta) - A Species Checklist for the Countries. - In: Blank, Schmidt \& Taeger, Recent Sawfly Research: Synthesis and Prospects, Goecke \& Evers, Keltern, pp. 399-504.

Koponen, M., Jussila, R. \& Vikberg, V. 2008 [2009]. Suomen loispistiäisluettelo (Hymenoptera, Parasitica). Osa 6. heimo Ichneumonidae, alaheimot Phrudinae, Mesochorinae, Metopiinae, Acaenitinae, Oxytorinae, Collyriinae, Orthopelmatinae, Orthocentrinae s. lat. ja Diplazontinae. - Sahlbergia 14:68-95.

Koponen, M., Jussila, R. \& Vikberg, V. 2009. Suomen loispistiäisluettelo (Hymenoptera, Parasitica). Osa 7. heimo Ichneumonidae, alaheimot Alomyinae ja Ichneumoninae. - Sahlbergia 15(2):13-48.

Deletions

Sirex atricornis Kjell. (= S. juvencus (L.)). Taeger et al. 2006:470.

Tenthredopsis arrogans (Kon.) (= T. friesei (Kon.)). Taeger, Blank \& Liston 2010 Zootaxa 2580:654.

Athalia cordatoides Priesn. (= A. circularis (Klug). ibid.:261.

Cladius difformis (Panz.) $\quad(=C$. pectinicornis (Geoffr.)). Taeger et al. 2006:416.

- comari Stein (=C. pectinicornis (Geoffr.)). ibid.:416.

Priophorus foveivaginatus Mal. $(=$ Cladius (Priophorus) brullei Dahlb.). ibid.:416.

Nematinus willigkiae Stein (=N. luteus (Panz.)). ibid.:432.

Pristiphora denudata Kon. (= P. pallidiventris (Fall.). Taeger et al. 2010:447.

Pteronidea virescens (Hart.) (= Nematus (Pteronidea) bergmanni Dahlb.). Taeger et al. 2006:432.

Pachynematus kirbyi (Dahlb.) (=P. clitellatus (Serv.)). ibid.:438.

- trisignatus (Först.) (= P. clitellatus (Serv.)). ibid.:438.

- angustatus Lindqv. (= P. smithae Ross). ibid.:439.

- sulcatus Bens. (= P. fallax (Serv.)). Taeger et al. 2010:436.

Amauronematus excellens Fors. (= A. puniceus (Christ)). Taeger et al. 2006:410.

Eupontania viminalis (L.) (f.d.). Vikberg \& Zinovjev 2006 Beitr. Entomol. 56:259.

Phyllocolpa coriacea (Bens.) $\quad(=$ P. leucaspis (Tischb.)). Kopelke 2007 Senckenb. biol. 87:153.

Dirophanes tegularis (Thoms.) $(=D$. muricifer (Holmgr.)). Schönitzer, Hower, Melzer \& Diller 2006 Mitt. Münch. Entomol. Ges. 95:109.

Centeterus elongator Berth. Recorded outside Finland. Koponen et al. 2009:18.
Diadromus arcticus Thoms. Recorded outside Finland. ibid.:22.

Nematomicrus tenellus Wesm. Recorded outside Finland. ibid.:23.

Pristicerops sternoleucus (Wesm.) (f.d., $=P$. infractorius (L.)). ibid.:24.

Platylabus pedatorius (F.) (f.d., $=$ P. heteromallus (Berth.)). ibid.:25.

- stolidus Perk. (f.d., = P. odiosus Perk.). ibid.:25.

- daemon (Wesm.) (f.d., = P. pallidens (Wesm.)). ibid.:25.

- zagoriensis Heinr. (=P. tricingulatus (Grav.)). Riedel 2008 Spixiana 31:156. Koponen et al. 2009:26.

- rufiventris Wesm. (= P. vibratorius (Thunb.)). Riedel 2008 Spixiana 31:158.

- pullus Wesm. Recorded outside Finland. Koponen et al. 2009:16, 25.

Anisobas hostilis (Grav.) (f.d., = A. rebellis Wesm.). Horstmann 2007 Entomofauna 28:109.

- jugorum Heinr. (= A. rebellis Wesm.). ibid.:108.

- parviceps (Thoms.) (=A. rebellis Wesm.). ibid.:108.

- seyrigi Heinr. (f.d.). ibid.:112.

Cratichneumon sahlbergi Hellén Recorded outside Finland. Koponen et al. 2009:28.

Virgichneumon mesostilpnus (Thoms.) $\quad(=\mathrm{V}$. albosignatus (Grav.)). ibid.:30.

Ichneumon caloscelis Wesm. Recorded outside Finland. ibid.:31.

- gravipes Wesm. (=I. melanosomus Wesm.). ibid.:33.

- incomptus Holmgr. (= I. memorator Wesm.). ibid.:34.

- macrocerophorus D.T. (=I. melanotis Holmgr.). ibid.:34.

Pseudoamblyteles homocerus (Wesm.) Recorded outside Finland. ibid.:36.

Exephanes femoralis Brischke (f.d., = E. occupator (Grav.)). ibid.:36.

Hoplismenus aulicus (Grav.) (f.d., = Hybophorellus injucundus (Wesm.)). ibid.:39.

Arotes albicinctus (Grav.) Recorded outside Finland. Koponen et al. 2008:78.

Cylloceria accusator (F.) (f.d., = C. melancholica (Grav.)). ibid.:79.

Stenomacrus confinis (Holmgr.) $(=S$. cognatus (Holmgr.). ibid.:86.

- vitripennis (Holmgr.) Recorded outside Finland. ibid.:87.

Plectiscus flavicornis (Thoms.) (= P. agilis (Holmgr.)). ibid.:86.

Eusterinx bispinosa (Strobl) (alpigena (Strobl)) (f.d., $=$ E. circaea Ross.). Humala et al. 2007 Sahlbergia 12:55.

Plectiscidea agitator (Först.) Recorded outside Finland. Koponen et al. 2008:83. 
Hemiphanes townesi Ross. (f.d., $=H$. performidatum Ross.). ibid.:82.

Lathrolestes abdominalis (Brsch.) (= L. verticalis (Brsch.) (marginatus Thoms.)). Horstmann 2005 Mitt. Münchn. Ent. Ges. 95:78.

Dusona adriaansei (Teun.) (=D. carpathica (Szépl.)). Horstmann 2009 Spixiana 32:47.

- debilis (Först.) (=D. flagellator (F.)). ibid.:48.

- subaequalis (Först.) (=D. rugifera (Först.)). ibid.:50.

Hyposoter neglectus (Holmgr.) (f.d., = Phobocampe variabilis Šed.) Šedivý 2004 Acta Univ. Carol., Biol. 48:232.

Cidaphus alarius (Grav.) (f.d., = C. areolatus (Boie)). Koponen et al. 2008:71.

Mesochorus fulvus Thoms. (= M. fulgurans Curt.). Horstmann 2007 Linz. biol. Beitr. 38:1462.

- stigmaticus Brischke (= M. pallipes Brischke). ibid.:1473.

Hypsicera flaviceps Ratz. Recorded outside Finland. Koponen et al. 2008:76.

Erigorgus rufus (Haberm.) (= Barylypa propugnator (Först.)). Schnee 2008 Beitr. Entomol. 58:252.

Microchelonus rimulosus (Thoms.) (= M. sulcatus (Jur.)). Tobias 2010 Tr. Russk. Entomol. Obšč. 81(1):30.

Acropiesta flavipes Kieff. (= A. sciarivora (Kieff.)). Macek 1998 Folia Heyrovskyana 6:26.

Zygota excisipes (Kieff.) (=Z. excisor (Zett.)). Macek 1997 Folia Heyrovskyana 5:44.

- norvegica (Kieff.) (=Z. ruficornis (Curt.)). ibid.:43.

Meuselia fuscicornis Kieff. (= Opazon parvulum (Hal.)). Macek 1995 Folia Heyrovskyana 3:82.

Trichopria elongata (Thoms.) (= Diapria nigricornis Thoms.). Notton 1995 Beitr. Entomol. 45:279.

Scelio thomsoni Kieff. (= S. inermis (Zett.). Kononova \& Kozlov 2008 Opred. faune Rossii 172:177.

Platygaster apicalis Thoms. (= P. pelias Walk.). Buhl \& Notton 2009 Journ. Nat. Hist. 43:1681.

The following species have by mistake been added twice to the list:

Dichrogaster heteropus (Thoms.) Silfverberg $1991 \mathrm{EF}$ 2:16 \& Silfverberg 1996 EF 7:47.

Charitopes areolaris (Thoms.) Hellén 1966 NE 46:84 \& Silfverberg 1986 NE 66:145.

Mastrus sordipes (Grav.) Hellén 1966 NE 46:84 \& Silfverberg 1986 NE 66:145.

Zoophthorus punctiventris (Thoms.) Hellén 1966 NE 46:84 \& Silfverberg 1986 NE 66:145.

Theroscopus ornaticornis (Schmied.) Hellén 1952 NE 32:78 \& Hellén 1971 NE 51:86.

Atractodes alutaceus Thoms. Hellén 1971 NE 51:86 \& Silfverberg 1981 NE 61:59.

Dicaelotus pusillator (Grav.) Hellén 1952 NE 51:77 \& Hellén 1961 NE 41:118.
Additions

Aprosthema bifidum (Klug) (sp.dist.). Taeger, Blank \& Liston 2010 Zootaxa 2580:161.

Arge fuscipes (Fall.) (sp. dist.) Finland. Taeger et al. 2006:405.

Heptamelus dahlbomi (Thoms.) (sp. dist.) Ab, N, Ta, $S a, K b$. Vikberg \& Liston 2009 Zootaxa 2112:8.

Pseudohemitaxonus sharpi (Cam.) Ta: Janakkala. Vikberg 2010 Sahlbergia 16:12.

Tenthredo temula Scop. Al, Sa. Paukkunen et al. 2009 Sahlbergia 15:18.

Macrophya teutona (Panz.) Ab, N, Ta. ibid.:18.

Harpiphorus lepidus (Klug) Ab: Turku Ruissalo (I. Kakko). Vikberg \& Vårdal 2010 Sahlbergia 16:8.

Claremontia puncticeps (Kon.) (sp. dist.). Taeger et al. 2010:318.

Cladius ulmi (L.) Ta: Ypäjä (I. Kakko). Paukkunen et al. 2009:19. Kakko 2009 Lounais-Hämeen Luonto 96:16.

Hoplocampa flava (L.) Sa, Kl. Paukkunen et al. 2009:19.

- fulvicornis (Panz.) Ab: Lohja. ibid.:19.

Eupontania brevicornis (Först.) Ab, Ta. Vikberg \& Zinovjev 2006:250.

- myrtilloidica (Kop.) Ok: Kuhmo \& Suomussalmi. ibid.: 254.

* ( - collectanea) rosmarinifoliae Vikb. \& Zin. Ab, $N$, Ta. ibid.:259.

* - acutifoliae baltica Vikb. \& Zin. N: Espoo. ibid.:260.

*Tubpontania nitidinota Vikb. Ab, N, St, Ta, Sa, Kl, Sb, Om, Ob. Vikberg 2010 Zootaxa 2610:20.

Odontocolon thomsoni (Clém.) Ta: Sahalahti (P. Valtonen). Jussila \& Várkonyi 2009 w-album 8:4.

Xorides indicatorius (Latr.) Ta: Sahalahti (P. Valtonen). Jussila 2007 w-album 3:4.

Acrolyta flagellator Schw. \& Sh. Ab, N, Ka. Jussila \& Várkonyi 2009:6.

- nigricolor (Aub.) Ab: Nousiainen, Ta: Lahti. ibid.:6.

Hemiteles maricesca Schw. \& Sh. Ab: Nousiainen. ibid.:6.

- rubropleuralis Kiss $A b$ : Turku. ibid.:6.

Dichrogaster liostylus (Thoms.) Ab, N. ibid.:7.

- modesta (Grav.) Ab, Ka, Ta. ibid.:7.

Gelis infumatus (Thoms.) Ab: Turku. ibid.:7.

- operosus Schw. Oa, Sb. ibid.:7.

- parens Schw. $N$ : Inkoo. ibid.:8.

- recens Schw. Sb: Savonranta, Ob: Tervola. ibid.:8.

- sanguinipectus (Schmied.) Ab: Sauvo. ibid.:8.

- spurius (Först.) Al, Ab, St, Ta, Sa, Oa, Tb, Sb, Ob, Li. ibid.:8.

- venatorius (Först.) $A b, N, T a, O a, T b, K b, L i$. ibid.:9.

Endasys strictogastris Saw. \& Luhm. Ab, Ta, Ok. Jussila 2010 w-album 9:7. 
- talitzkii (Tel.) Ab: Taivassalo (A. Haarto \& V.-M. Mukkala). ibid.:8.

Bathythrix margaretae Saw. Ab, Sb. ibid.:8.

- prominens (Stroblo) $A b, K b$. ibid.:8.

Sulcarius pubescens Town. Ta: Sahalahti (P. Valtonen). Jussila \& Varkonyi 2009:9.

Oresbius bipunctatus (Schmied.) Ob: Tervola (P. Martikainen). ibid.:10.

Pleolophus vestigialis (Först.) Ab, N, Ka, Ta, Sa. ibid.: 10 .

Plectocryptus poecilops (Kriechb.) $N$ : Pyhtää, $K a$ : Kotka. Jussila 2010:8.

Heterischnus nigricollis (Wesm.) (sp.dist.) Koponen et al. 2009:17.

Oronotus binotatus (Grav.) N: Helsinki (O. Ranin). ibid.:15.

Eriplatys sawoniewiczi Dill. Ab: Turku (R. Jussila). ibid.: 15 .

Tycherus dodecellae Ranin N: Helsinki (O. Ranin). ibid.:15.

- vagus (Berth.) N: Helsinki (O. Ranin), Hyvinkää (M. Koponen), ibid.:15.

Phaeogenes trepidus Wesm. Ab: Vihti (V.J. Karvonen), ibid.:15.

Diadromus collaris (Grav.) N: Helsinki (O. Ranin). ibid.: 15 .

- intermedius Wesm. Ab, N. ibid.:15.

Aethecerus exilis Wesm. Ta: Forssa (M. Nylund). ibid.:15.

Trachyarus anceps (Berth.) (specularis Rom.) (sp.dist.). Gokhman 2007 Spixiana 30:66.

* - borealis Zwakh. Tb: Jyväskylä (J.A. Elzinga). Zwakhals \& Elzinga 2009 Mitt. Münch. Entomol. Ges. 99:129.

- solyanikovi Gokhm. Tb: Jyväskylä. ibid.:192.

- brevipennis Rom. Sa, Tb. Koponen et al. 2009:15.

Linycus flavitarsis (Heinr.) N, St, Ta, Oa. ibid.:15.

Cyclolabus alpinus (Hab.) Tb: Pihtipudas (A. Saarinen). ibid.:15.

Asthenolabus stellae (Heinr.) Ta: Iitti (R. Teriaho). ibid.:15.

Anisobas rebellis Wesm. (sp. dist.). Finland. Horstmann 2007 Entomofauna 28:108.

Pristicerops larvator (Grav.) Finland. Koponen et al. 2009:24.

* Platylabus baueri Ried. N: Hanko Russarö (A. \& J. Kullberg). Riedel 2008 Spixiana 31:121.

* -fennicus Ried. N: Pärnå ( $\AA$. Nordström). ibid.:128.

- heteromallus (Berth.) (pedatorius auct.) "Finnland". ibid.:131. Koponen et al. 2009:25.

* - minor Ried. Al, Ab, Ta, Oa. Riedel 2008:137.

- neglectus Fonsc. "Finnland". ibid.:138.

- obator (Desv.) "Finnland". ibid.:140.

- oehlkei Heinr. "Finnland". ibid.:142.

- orbitalis (Grav.) "Finnland". ibid.:144.
- perexiguus Heinr. "Finnland". ibid.:146.

* - pseudopumilio Ried. Ab: Turku \& Sauvo (R. Jussila). ibid.:148.

* - rufocoxatus Ried. Al, Ab, N, Sa, Kl. ibid.:150.

- pallidens Wesm. (daemon auct.) Koponen et al. 2009:25.

Pseudoplatylabus violentus (Grav.) Ab: Turku (R. Jussila). ibid.:15.

Cratichneumon dissimilis (Grav.) Finland. ibid.:27.

Virgichneumon maculicauda (Perk.) Ta: Tampere (T. Grönblom). ibid.:15.

Barichneumon plagiarius (Wesm.) Ab: Sauvo (R. Jussila). ibid.:15.

Ichneumon analisorius Heinr. Sa: Hirvensalmi (O. Ranin). ibid.:16.

- eremitatorius Zett. Finland. ibid.:32.

- hypolius Thoms. Sa: Ruokolahti (W. Hellén). ibid.:16.

- leucopeltis Thoms. Al: Lemland (W. Hellén). :16.

- pseudocaloscelis Heinr. Sa: Hirvensalmi (O. Ranin). ibid.:16.

- stramentor Rasn. $N$, Ta, Sb. ibid.:16.

Exephanes ischioxanthus (Grav.) Finland. ibid.:36.

Syspasis scutellator (Grav.) Ab, N, Ka, Ta, Sa, Ob, Tb, $\mathrm{Kb}, \mathrm{Om}$. Koponen, Vainio, Vikberg \& Jussila 2008 Sahlbergia 13(2):41.

Lymantrichneumon disparis (Poda) Finland. Koponen et al. 2009:39.

Coelichneumon atratorius (Vill.) Ka: Kotka (L. Luukkonen). ibid.:16.

- biguttorius (Thunb.) Ab: Houtskär (R. Jussila), Sauvo (R. Jussila). ibid.:16.

Mesoclistus rufipes (Grav.) Le: Kilpisjärvi (V.J. Karvonen). Koponen et al. 2008:70.

Enizemum nigricorne (Thoms.) $\mathrm{Al}, \mathrm{Ab}, \mathrm{N}, \mathrm{Ka}, \mathrm{Ta}$. Jussila 2007:8.

Campocraspedon annulitarsis (Hedw.) N: Sipoo (N. Laurenne). Koponen et al. 2008:87. Jussila \& Várkonyi 2009:5.

Syrphophilus asperatus Dasch Le: Enontekiö. Jussila 2009 Metsähall. luonnons.julk. A 185:45.

Bioblapsis polita (Snell.) Al, Ab, St, Ta, Oa. Jussila 2007:7.

Sussaba aciculata (Ruthe) Le: Enontekiö. Jussila 2009:45.

Aperileptus melanopsis Först. Finland. Humala 2003:112. Koponen et al. 2008:80.

- tricinctus Först. Finland. Humala 2003:113.

Orthocentrus corrugatus Holmgr. Finland. Koponen et al. 2008:84.

- longicornis Holmgr. Ab: Vihti, Lk: Kolari. ibid.:70.

- strigatus Holmgr. N: Vantaa (O. Ranin). ibid.:70.

Picrostigeus antennalis Rom. Finland. ibid.:85.

- debilis (Grav.) Al, Ab, N, Oa, Sb, Ob. Jussila 2007:6. 
* - svecofennicus Juss. Le: Saana (B. Federley), Li: Utsjoki Kevo (R. Jussila). ibid.:6.

Stenomacrus deletus (Thoms.) Ab, N, Oa. ibid.:5.

- molestus (Holmgr.) Al, Ab, Le, Li. Koponen et al. 2008:70.

- vafer (Holmgr.) Al, Ab, Ta, Le, Li. ibid.:70.

Plectiscus exilis (Holmgr.) Li: Utsjoki. Jussila 2007:5.

Gnathochorisis flavipes Först. Finland. Humala 2003:140.

Eusterinx inaequalis Ross. $A b, N, K l, T b, K b$. Humala, Jussila \& Koponen 2007 Sahlbergia 12:54.

- minima (Strobl) Ab, Tb, Le, Li. ibid.:54.

- obscurella Först. Finland. Humala 2003:143.

- pseudoligomera Greg. Finland. ibid.:144

- refractaria Ross. Ab, N, Ka, St, Tb, Kb, Li. Humala et al. 2007:54.

- jugorum (Strobl) Ab: Nystad (W. Hellén), Ks: Kuusamo (J. Viramo). Koponen et al. 2008:70.

* - fennoscandica Hum. N: Nurmijärvi \& Orimattila (M. Koponen). Humala 2008 EF 19:101.

*Fennomacrus koponeni Hum. Sa: Ristiina (M. Koponen). Humala 2003:130 (Plectiscidea k.). Humala 2008:96.

*Plectiscidea aquilonia Hum. Ab: Turku (M. Koponen). Humala 2003:130. Humala 2008:97.

- bistriata (Thoms.) Finland. Humala 2003:130.

- communis (Först.) Finland. ibid.:135.

- erythropyga (Först.) Finland. ibid.:131.

- nemorensis Ross. Ab, N, St, Sa. Humala et al. 2007:57.

- posticata (Först.) Ab, N, Kb. ibid.:57.

- undulata (Dasch) Ta: [Ruovesi] Multinharju (M. Kuussaari \& J. Siitonen). ibid.:57.

* - fuscifemur Hum. Ab, N. Humala 2003:130. Humala 2008:99.

* - helleni Hum. Le: Kilpisjärvi (W. Hellén). Humala 2003:131. Humala 2008:100.

Proclitus ardentis Ross. $A b, N, T a, T b, K b, O b$. ibid.:56.

- edwardsi Rom. N: Vantaa (V. Karvonen). ibid.:56.

Pantisarthrus dispar Ross. Finland. Humala 2003:104.

- gracilis Ross. Finland. ibid.:104.

Aniseres caudatus Hum. Ab, $N, K b$. Humala et al. 2007:52.

- subarcticus Hum. Ab, Sa, Kb. ibid.:52.

Helictes carinalis Hum. N: Åggelby (T. Clayhills). ibid.:56.

Megastylus excubitor (Först.) Finland. Koponen et al. 2008:82.

Scambus alpestrator Aub. Le: Enontekiö. Jussila 2009:44. Jussila 2010:3.

*Zatypota kerstinae Fritzén $A b, O a, S b, K b$. Fritzén 2010 Zootaxa 2487:53.
Pseudorhyssa nigricornis (Ratz.) Ka: Kotka (L. Luukkonen). Jussila 2007:3.

Collyria trichophthalma (Thoms.) Ta: Janakkala. Koponen et al. 2008:70.

Microleptes rectangulus (Thoms.) Ab: Vihti (V. Karvonen). Humala et al. 2007:51.

Orthopelma brevicorne Morl. Ab: Houtskär \& Korpo (R. Jussila). ibid.:58.

Glypta longicauda Hart. Ab: Turku. Jussila 2010:4.

Lissonota versicolor Holmgr. Ab: Parainen (M. Vuola). ibid.:4.

- inareolata Pfeff. $A b$ : Korpo Utö. Jussila \& Várkonyi 2009:4.

Hodostates brevis (Thoms.) Ok: Kuhmo. ibid.:4.

Priopoda xanthopsana (Grav.) N: Siuntio. Jussila 2010:4.

Lathrolestes albicinctus (Haberm.) Ab: Turku. ibid.:4.

Neostroblia longigena (Thoms.) Sb: Varkaus (N. Laurenne). Jussila \& Várkonyi 2009:4.

* Campodorus suomi Kasp. Le: Kilpisjärvi (R. Hinz). Kasparyan 2006 Entomol. Obozr. 85:646.

- infidus (Woldst.) Le: Enontekiö. Jussila 2009:44. Jussila 2010:5.

Saotis albionis Kasp. N: [Helsingfors] Munksnäs (E. Lindqvist). Kasparyan \& Kopelke 2010 Entomol. Obozr. 89:249.

* - boreator Kasp. \& Kop. Ta: Urjala (T. Reilin). ibid.:251.

* - granulator Kasp. \& Kop. Sb: Nilsiä (R. Jussila). ibid.:254.

* - subarctor Kasp. \& Kop. Le: Kilpisjärvi (J.-P. Kopelke), $L i$ : Ivalo (W. Hellén). ibid.:260.

Phobetes latipes Thoms. (sp. dist.) Finland. Horstmann 2007 Mitt. Münchn, Ent. Ges. 97:77.

Euryproctus mundus (Grav.) Sb: Varkaus (N. Laurenne). Jussila \& Várkonyi 2009:5.

Barycnemis alpina (Strobl) Ok: Kuhmo, Li: Utsjoki. Jussila 2007:5

- gravipes (Grav.) Al, Ab, N, Oa, Li. ibid.:5.

*Phobocampe punctata Šed. Ks: Salla (K. Ruohomäki). Šedivý 2004 Acta Univ. Carol., Biol. 48:228; Jussila 2007:4.

- tempestiva (Holmgr.) Al, Ab, Ka, Tb, Li. Jussila 2007:4.

* - variabilis Šed. (neglecta auct.) Finland. Šedivý 2004:232.

Diadegma velox (Holmgr.) Ab: Nousiainen. Jussila \& Várkonyi 2009:5.

Hyposoter clausus (Brischke) Ta: Sahalahti (P. Valtonen). ibid.:5.

Astiphromma hamulum (Thoms.) Li: Utsjoki (V. Karvonen). Koponen et al. 2008:69. Jussila 2010:5.

- pictum (Brischke) $S b$ : Leppävirta (E. Tiihonen). Koponen et al. 2008:69. 
- striatum (Brischke) Ab, N, Tb, Le, Li. ibid.:69.

- albitarse (Brischke) Ab, N, Ka, Ta, Oa. Jussila 2010:5.

- luridum Schwenke Ta: Somero (T. Mutanen). ibid.:5.

- rimosum Schwenke Sa: Mikkeli (M. Koponen). ibid.:6.

Mesochorus curvicauda Thoms. Ab, $N$, Ka. Koponen et al. 2008:69.

- femoralis Brischke $N, \mathrm{Ka}, \mathrm{Sa}$, Li. ibid.:69.

- fuscicornis Brischke $A b, N$. ibid.:69.

- petiolaris Brischke Ab, N, Ka, Li. ibid.:69.

- plumosus Dasch Ta: Janakkala (V. Vikberg). ibid.:70.

- zygaenae Schwenke Ks: Kuusamo (R. Jussila). ibid.:70.

- testaceus Grav. [Schwenke 1999] ibid.:74.

- alpigenus Strobl Le: Enontekiö. Jussila 2009:45.

Stictopisthus bilineatus (Thoms.) N: Vantaa (V. Karvonen). Jussila 2010:7.

- flavescens (Fonsc.) N, St. ibid.:7.

- tenuigaster Schwenke $A b$ : Sauvo. ibid.:7.

Trieces facialis (Thoms.) Ab, N, Ta, Sa, Kb, Om, Ok. Koponen et al. 2008:70.

Triclistus pygmaeus (Cress.) Finland. ibid.:76.

Exochus fletcheri Bridgm. N: Kirkkonummi (V.J. Karvonen). ibid.:70.

- quadrimaculatus Schmied. Ab: Vihti (V.J. Karvonen). ibid.:70.

- separandus Schmied. Ab: Vihti, N: Kirkkonummi. ibid.:70.

- thomsoni Schmied. Ab: Naantali, N. Helsinki. ibid.:70.

Bracon marshalli Szépl. Finland. Papp 2008 Linzer biol. Beitr. 40:1764.

- subsinuatus Szépl. Finland. ibid.:1782.

- curticaudis Szépl. Finland. ibid.:1753.

*Colastes fritzeni Acht. \& Shaw Ta: Valkeakoski (N. Fritzén \& I. Österblad), Oa: Korsholm (I. Österblad), Om: Lohtaja (N. Fritzén). van Achterberg \& Shaw 2008 Journ. Nat. Hist. 42:1854.

Orgilus obesus Taeg. Ok: Vaala. Leinonen \& Itämies 2008 Baptria 33(3):10.

Microchelonus labipalpis Tob. Finland. Tobias 2010

Tr. Russk. Entomol. Obšč. 81(1):52.

- fischeri Tob. Finland. ibid.:109.

- laplandicus Tob. Finland. ibid.:186.

Neuroterus anthracinus (Curt.) Ab: Korpo Vattkast. Tack 2010 Diss., p. 52.

- albipes (Schr.) Al: Eckerö \& Lemland. Kaartinen \& Janhonen 2010 Memoranda Soc. Fauna Flora Fenn. 85:98.

Andricus pseudoinflator Tav. Al: Eckerö, Finström \& Lemland. ibid.:98.

- seminationis (Gir.) Al: Föglö. ibid.:98.

- testaceipes Hart. Al: Föglö. ibid.:98.
Eupelmus fuscipennis Först. Ab: Pojo (E. Vuorinen). Vikberg 2008 [2009] Sahlbergia 14:60.

Cirrospilus diallus Walk. Ab: Korpo Vattkast. Tack 2010:42.

- lyncus Walk. Ab: Korpo Vattkast. ibid.:42.

Achrysocharoides butus (Walk.) Ab: Korpo Vattkast. ibid.:42.

Palaeomymar anomalus (Bl. \& Kr.) Ab: Karkkila, Ta: Janakkala. Vikberg \& Gergely 2006 Sahlbergia 11(2):42.

Zygota comitans Macek Li: Utsjoki Kevo. Macek 1997 Folia Heyrovskyana 5:47.

Dryinus niger Kieff. St: Eurajoki, Sa: Mikkeli. Väänänen \& Vikberg 2008 Sahlbergia 13(2):2.

Gonatopus solidus (Haupt) N: Hanko (J. Perkiömäki). ibid.:3.

Chrysis corusca Valk. Sa: Lappeenranta (M. Raekunnas). Paukkunen et al. 2009 Sahlbergia 15:6.

- insperata Chevr. Ka: Virolahti (Y. Ranta). ibid.:6.

- leptomandibularis Nieh. Sb: Vehmersalmi (D. Hemdal). ibid.:6.

- mediata Linsenm. Ab: Karjalohja (U. Saalas), $N$ : Kirkkonummi (P. Haglund). ibid.:6.

Myrmica vandeli Bondr. Ab: Lohja (O. Engström). ibid.:7.

Lasius sabularum (Bondr.) Ab: Turku (M. Saaristo), $K b$ : Pielisjärvi (P. Lehtinen). ibid.:7.

Formica clara Forel (lusatica Seif.) Ks. ibid.:7.

Arachnospila alvarabnormis Wolf N: Hanko (S. Karjalainen \& L. Pulli). ibid.:8.

Psenulus schencki (Tourn.) Ab: Turku (I. Kakko). ibid.:8.

Pemphredon mortifer Valk. Sa: Joutseno (E. Thuneberg). ibid.:8.

Crabro ingricus (Moraw.) Ks: Kuusamo (K. Mattila). ibid.:8.

Hylaeus paulus Bridw. N: Inkoo. ibid.:9.

Andrena bluethgeni Stoeckh. Al: Maarianhamina (M. Raekunnas), Sa: Lappeenranta (A. Valtonen \& J. Jantunen). ibid.:9.

Halictus leucaheneus Ebm. Sa: Valkeala (A. Valtonen \& J. Jantunen). ibid.:11.

Osmia bicornis (L.) (rufa (L.)) Sa: Imatra \& Lappeenranta. Saarinen 2006 Suomen Luonto 65(6): 16. Paukkunen et al. 2009:13.

- bicolor (Schr.) N: Inkoo (P. Malinen). Paukkunen et al. 2009:13.

- svenssoni Tkalcu Le: Enontekiö. ibid.:14.

Coelioxys alata Först. N: Siuntio (J. Pöyry), Sa: Mikkeli (J. Pöyry). ibid.:14.

Nomada ferruginata (L.) N: Helsinki \& Porvoo. ibid.: 15 .

- succincta Panz. N: Vantaa (T. Salin). ibid.:17. 
Table 1. Number of insect species of different orders reported at different times.

\begin{tabular}{lrrrrr}
\hline & 1950 & 1975 & 2000 & 2005 & 2010 \\
\hline Collembola & 156 & 170 & 218 & 215 & 242 \\
Diplura & 1 & 1 & 1 & 1 & 1 \\
Protura & 1 & 3 & 3 & 3 & 3 \\
Thysanura & 4 & 4 & 5 & 5 & 5 \\
Ephemeroptera & 57 & 49 & 51 & 55 & 55 \\
Odonata & 50 & 51 & 52 & 54 & 57 \\
Plecoptera & 35 & 35 & 35 & 35 & 35 \\
Dermaptera & 2 & 2 & 2 & 2 & 2 \\
Orthoptera & 35 & 36 & 32 & 32 & 32 \\
Dictyoptera & 6 & 6 & 7 & 7 & 7 \\
Psocoptera & 51 & 57 & 62 & 67 & 71 \\
Phthiraptera & 147 & 223 & 292 & 294 & 294 \\
Hemiptera & 902 & 1,148 & 1,359 & 1,428 & 1,549 \\
Thysanoptera & 113 & 113 & 114 & 118 & 139 \\
Neuroptera & 61 & 62 & 62 & 63 & 66 \\
Coleoptera & 3,239 & 3,472 & 3,629 & 3,657 & 3,700 \\
Strepsiptera & 6 & 7 & 5 & 5 & 5 \\
Trichoptera & 202 & 202 & 205 & 215 & 216 \\
Lepidoptera & 2,071 & 2,240 & 2,378 & 2,509 & 2,558 \\
Mecoptera & 6 & 6 & 6 & 6 & 6 \\
Diptera & 4,098 & 4,782 & 5,237 & 5,550 & 6,212 \\
Siphonaptera & 44 & 45 & 52 & 52 & 54 \\
Hymenoptera & 3,700 & 4,962 & 5,975 & 6,158 & 6,304 \\
\hline Total & 14,987 & 17,752 & 19,549 & 20,516 & 21,613 \\
\hline
\end{tabular}

Comments

Humala (2003) described three new Plectiscidea-species from Finland ( $P$. fuscifemur, $P$. helleni and $P$. koponeni), Helictes carinata from Finland and the Far East, and Helictes karelica from Finland and Karelia, but he did not include any type designations for those species. Those names were therefore unavailable according to the present Code of Nomenclature (Art. 16.4). In 2008 the Plectiscidea species were described in an appropriate way.

Present number: $6,158-73+219=6,304$ spp.
Table 1 shows how the number of reported species has changed in the different insect orders since 1950. This might give some indication on potential future development. 